\title{
Liste annotée des Diptères Chironomidés de France continentale et de Corse
}

\author{
B. Serra-Tosio! \\ H. Laville2
}

Mots clés : Diptera, Chironomidae, faunistique, France.

Les auteurs présentent la première liste des Diptères Chironomidae signalés jusqu'en 1990 de France continentale et de Corse. Il a été tenu compte des synonymies anciennes et récentes. Des corrections et des additifs sont apportés aux listes régionales publiées antérieurement. La présentation choisie permet de faire le lien avec la partie consacrée aux Chironomidés dans la Limnofauna Europaea éditée par Illies (1978). Avec 598 espèces recensées, le territoire français s'avère être l'un des plus riches d'Europe en Diptères Chironomidés.

\section{An annotated check list of the Diptera Chironomidae occurring in continental France and Corsica}

Keywords : Diptera, Chironomidae, faunistic, France.

A check list is provided for the first time for the Chironomidae (Diptera) recorded in continental France and Corsica up to 1990. The list includes recent and old synonyms. Some corrections and additions are added to regional lists previously published. The presentation facilitates a connection with the section dealing with Chironomid distribution in the Limnofauna Europaea (Illies 1978). With 598 recently recorded species, French territory is probably one of the richest areas of Europe for Chironomids.

\section{Introduction}

Afin de faire le bilan de nos connaissances concernant les Diptères Chironomidés de France, trois inventaires faunistiques ont été publiés depuis 1986 qui intéressent plusieurs aires biogéographiques de la Limnofauna Europaea (Fittkau \& Reiss 1978) dans lesquelles ces insectes avaient fait l'objet de recherches les plus intensives.

Ainsi 288 espèces ont été recensées dans les Pyrénées françaises (Laville \& Vinçon 1986), 261 espèces dans le Massif Central (Laville \& Serra-Tosio 1987), 335 espèces dans les Alpes françaises (SerraTosio $1989 \mathrm{~b}$ ), où s'interpénètrent les zones 2 et 13 de la Limnofauna pour la première, les zones 8 et 13 pour la deuxième, les zones 4 et 13 pour la troisième.

1. Laboratoire de Biologie alpine (Zoologie et Hydrobiologie), Université Joseph Fourier (Grenoble 1), B.P. 53 X, F-38041 Grenoble Cédex France.

2. Laboratoire d'Hydrobiologie, U.A. 695 C.N.R.S., Univerjité Paul Sabatier, 118, route de Narbonne, F-31062 Toulouse Cedex France.
Au total, 496 espèces ont été répertoriées dans ces trois régions essentiellement montagneuses du sud de la Loire et du sud du Rhône en amont de Lyon, soit à peu près la moitié méridionale de la France.

Restaient à recenser les grandes plaines occidentales (zone 13), les massifs de moyenne montagne des Vosges et du Jura français (zones 8 et 9) et la Corse (zone 3).

Ces régions, à l'exception d'un inventaire sur le bassin du Doubs dans le Jura français (Verneaux \& Vergon 1974), ont été moins systématiquement prospectées et n'ont fait l'objet que d'études plus fragmentaires et, dans l'ensemble, plutôt anciennes, notamment par Kieffer (1898-1925), Goetghebuer (1927-1944) et Gouin (1936-1957).

La faune de France de Goetghebuer (1927, 1928, 1932) n'est en fait qu'une extrapolation de la faune du nord de l'Europe puisqu'elle réunit essentiellement des espèces d'Allemagne, d'Angleterre, de Belgique et de Scandinavie : seulement 60 espèces ont été récoltées réellement sur le territoire français. 
Ce travail s'inscrit dans la même ligne que les précédents articles sur les Pyrénées, le Massif Central et les Alpes; il présente le premier bilan exhaustif, sauf erreurs ou omissions, de la faune des Diptères Chironomides de France avec la localisation de leurs aires de récoltes.

Pour cette synthèse, près de 74 travaux supplémentaires parus depuis le début du siècle jusqu'en 1990 ont été analysés.

Cette analyse a été actualisée conformément à la terminologie du Catalogue des Chironomidae paléarctiques (Ashe \& Cranston 1990 - dépôt manuscrit décer Jre 1982) où 178 genres et 1290 espèces sont répertoriés avec changements de nomenclature, synonymies et nombreux nomina dubia.

Des corrections et des additifs ont été apportés aux listes régionales publiées antérieurement qui tiennent compte également des plus récentes et importantes révisions systématiques parues entre 1982 et 1990, notamment celle de Saether (1990) concernant le genre Limnophyes.

\section{Découpage de la France}

Le territoire français a été subdivisé en 6 aires (cf. fig. 1).

1. Aire A : correspond au nord-ouest de la France, et plus précisément aux départements suivants : Pas-de-Calais, Nord, Somme, Aisne, Seine-Maritime, Oise, Manche, Calvados, Eure, départements de la région parisienne, Seine-et-Marne, Orne, Eure-et-Loir, Finistère, Côtes-d'Armor (autrefois Côtes-duNord), Morbihan, Ille-et-Vilaine, Mayenne, Sarthe.

2. Aire B : correspond au nord-est de la France, et plus précisément aux départements suivants : Ardennes, Marne, Meuse, Meurthe-etMoselle, Moselle, Bas-Rhin, Aube, HauteMarne, Vosges, Haut-Rhin, Yonne, Côted'Or, Haute-Saône, Belfort, Saône-et-Loire, Jura, Doubs, Rhône, Ain.

Cette aire inclut notamment les Vosges et le Jura français.

3. Aire $C$ : correspond au centre de la France, et plus précisément aux départements suivants : Loire-Atlantique, Maine-et-Loire, Indre-etLoire, Loir-et-Cher, Loiret, Nièvre, Allier, Loire, Vendée, Deux-Sèvres, Vienne, Indre,
Cher, Charente-Maritime, Charente, HauteVienne, Creuse, Puy-de-Dôme, Gironde, Dordogne, Corrèze, Cantal, Haute-Loire, Ardèche, Landes, Lot-et-Garonne, Lot, Aveyron, Lozère, Gers, Tarn-et-Garonne, Tarn, Hérault, Gard, Aude.

Cette aire coincide avec ce que Laville et SerraTosio (1987) définissent comme « Massif Central et basses régions avoisinantes $»$.

4. Aire D : correspond aux départements français incluant les Pyrénées: PyrénéesAtlantiques (autrefois Basses-Pyrénées), Hautes-Pyrénées, Haute-Garonne, Ariège, Pyrénées-Orientales.

Cette aire coincide, en partie seulement, avec les Pyrénées de Laville (1980a ; 1980b) et de Laville et Vinçon (1987), car elle est limitée au versant français de la chaîne pyrénéenne et aux basses régions avoisinantes.

5. Aire $\mathrm{E}$ : correspond aux départements français incluant la chaîne des Alpes : Haute-Savoie, Savoie, Isère, Drôme, Hautes-Alpes, Alpes-deHaute-Provence (autrefois Basses-Alpes), Vaucluse, Bouches-du-Rhône, Var, AlpesMaltimes.

Cette uirc coincide exactement avec ce que Serra-Tosio (1989b) appelle « Alpes et basses régions avoisinantes », c'est-à-dire toute la partie du territoire français sit uée au sud et à l'est du Rhône, y compris la Camargue et le littoral de la Provence.

6. Aire $\mathrm{F}$ : correspond aux départements de la Corse : Haute-Corse et Corse-du-Sud.

\section{Rapports du découpage avec les régions de la Limnofauna Europaea}

Le découpage ci-dessus est arbitraire, mais pas totalement. Il a en effet été défini de façon, autant que faire se peut, à permettre la relation avec le découpage en régions de la Limnofauna Europaea d'Illies (1978) (que nous abrègerons en « Limnofauna » dans la suite du texte).

Ainsi (fig. 1) :

- l'aire $\mathbf{A}$ est située tout entière dans la région 13 de la Limnofauna. 


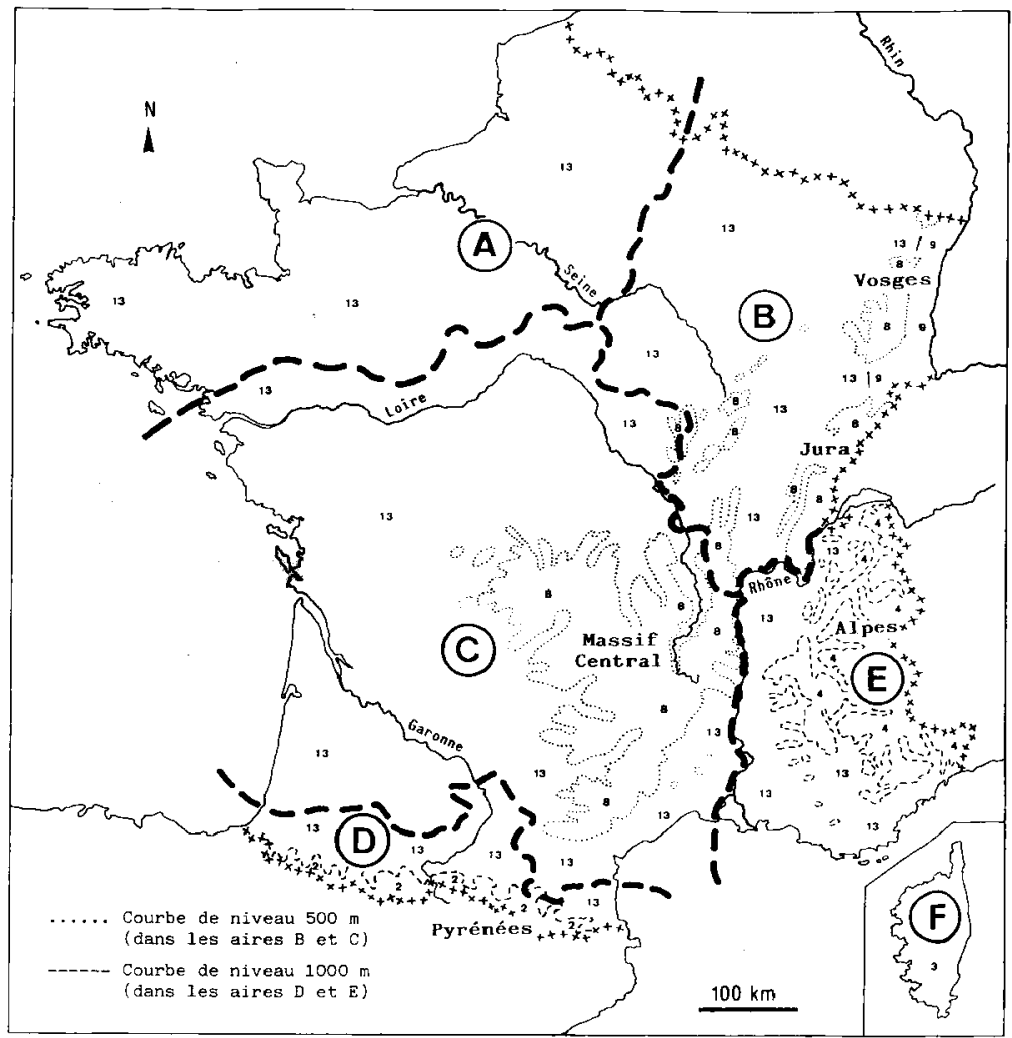

Fig. 1. Découpage de la France : aire A (Bretagne, Normandie, nord de la France) ; aire B (est de la France, Lorraine, Alsace, Jura) ; aire $C$ (Massif Central et basses régions avoisinantes); aire D (Pyrénées et basses régions avoisinantes) ; aire $E$ (Alpes et basses régions avoișinantes) ; aire $\mathrm{F}$ (Corse).

Les chiffres se rapportent aux régions de la Limnofauna Europaea. 
- l'aire B intéresse à la fois la région 8 de la Limnofauna, pour toutes les localités à altitude supérieure à $500 \mathrm{~m}$, et les régions 9 et 13 pour celles dont l'altitude est inférieure à $500 \mathrm{~m}$ (région 9 à l'est, c'est-à-dire la plaine d' $\mathrm{Al}$ sace ; région 13 à l'ouest et au nord).

- l'aire $\mathrm{C}$ intéresse à la fois la région 8 de la Limnofauna, pour toutes les localités à altitude supérieure à $500 \mathrm{~m}$, et la région 13 pour celles dont l'aititude est inférieure à $500 \mathrm{~m}$.

- l'aire $\mathrm{D}$ intéresse à la fois la région 2 de la Limnofauna, pour toutes les localités à altitude supérieure à $1000 \mathrm{~m}$, et la région 13 pour celles dont l'altitude est inférieure à $1000 \mathrm{~m}$.

- l'aire E intéresse à la fois la région 4 de la Limnofauna, pour toutes les localités à altitude supérieure à $1000 \mathrm{~m}$, et la région 13 pour celles dont l'altitude est inféricure à $1000 \mathrm{~m}$.

- l'aire $F$ est située dans la région 3 de la Limnofauna.

On constate donc que le territoire français envisagé ici constitue un ensemble biogéographique complexe et diversifié qui comprend une partie des régions 2, 3, 4, 8, 9 et 13 de la Limnofauna.

En conséquence, dans la liste des espèces de Chironomidés signalées de France présentée ci-après et pour permettre la correspondance entre notre découpage et celui de la Limnofauna, nous indiquons, pour chaque espèce, la lettre correspondant aux aires définies par nous, immédiatement suivie par le numéro de la région de la Limnofauna concernée. Le code est le suivant :
A 13 : aire $A$ (située tout entière dans la région 13 de la Limnofauna).
B 8: partie de l'aire $B$ située dans la région 8 de la Limnofauna (donc au-dessus de $500 \mathrm{~m}$ d'altitude).

B 9 : partie de l'aire $B$ située dans la région 9 de la Limnofauna (c'est-à-dire plaine du Rhin au-dessous de $500 \mathrm{~m}$ d'altitude).

B 13 : partie de l'aire $B$ située dans la région 13 de la Limnofauna (donc au-dessous de $500 \mathrm{~m}$ d'altitude, à l'exclusion de la plaine du Rhin).

C 8 : partie de l'aire $C$ située dans la région 8 de la Limnofauna (donc au-dessus de $500 \mathrm{~m}$ d'altitude).
C 13 : partie de l'aire $C$ située dans la région $13 \mathrm{de}$ la Limnofauna (donc au-dessous de $500 \mathrm{~m}$ d'altitude).

D 2 : partie de l'aire $D$ située dans la région 2 de la Limnofauna (donc au-dessus de $1000 \mathrm{~m}$ d'altitude).

D 13 : partie de l'aire $D$ située dans la région 13 de la Limnofauna (donc au-dessous de $1000 \mathrm{nl}$ d'altitude).

E 4 : partie de l'aire $E$ située dans la région 4 de la Limnofauna (donc au-dessus de $1000 \mathrm{~m}$ d'altitude).

E 13 : partie de l'aire E située dans la région $13 \mathrm{~d}$ la Limnofauna (donc au-dessous de 1000 is d'altitude).

F 3 : aire $F$ (située tout entière dans la région 3 de la Limnofauna).

\section{Remarques}

1) Dans le cas des aires B, C, D ou E, une localisation sans nombre de référence à la Limnofauna (par exemple $B$ ou $E$, etc.) résulte d'une absence d'informations sur l'altitude des stations signalées. Il nous est alors impossible de faire concorder notre découpage avec celui de la Limnofauna.

2) Beaucoup d'auteurs, en particulier des auteurs anciens (comme bien souvent Kieffer ou Gouin pour la Lorraine et l'Alsace, ou d'autres pour les Alpes), ont omis d'indiquer l'altitude des stations signalées. Dans de nombreux cas néanmoins, nous avons pu, par référence aux cartes et aux atlas, situer par rapport aux altitudes $500 \mathrm{~m}$ ou $1000 \mathrm{~m}$ les localités concernées et, par conséquent, préciser la région de la Limnofauna dont elles font partie.

De plus, en ce qui concerne la liste des Chironomidés du Jura (aire B) établie par Verneaux \& Vergon (1974), nous avons bénéficié de l'ajde du premier auteur (Verneaux, in litt.). Il nous a aimablement précisé l'amplitude de répartition verticale de la plupart des espèces trouvées dans ce massif, nous permettant ainsi le choix entre les régions 8 et 13 de la Limnofauna, alors que l'absence d'indications d'altitudes de la liste publiée initialement interdisait a priori toute distinction de cette sorte.

3) Dans la liste ci-jointe, après l'indication de présence dans les aires A, B ou F, on trouvera entre parenthèses les numéros des publications (cf. bibliographie) qui signalent l'espèce considérée de France. 
Un point d'interrogation (?) placé avant le numéro d'une publication signifie que l'auteur concerné estime l'identification de l'espèce comme incertaine, ou qu'il a fait précéder dans sa publication le nom du groupe-espèce par un point d'interrogation (?), ou par cf., ou par gr., ou par aff., etc., c'est-à-dire qu'il ne peut pas rapporter ce taxon à une espèce précise.

En revanche, pour les aires $C, D$ et $E$, les références bibliographiques ne sont pas indiquées ici (sauf erreurs ou omissions précisées en notes), car elles ont été données respectivement dans les publications suivantes : Laville \& Serra-Tosio (1987), Laville \& Vinçon (1986) et Serra-Tosio (1989b).

4) Dans l'aire $D$, certaines espèces citées par Laville (1980a) et Laville \& Vinçon (1986) sont connues du versant espagnol des Pyrénées, mais pas du versant français. Elles ont par conséquent été retirées de l'aire $\mathrm{D}$ dans la présente liste, puisque cette dernière ne concerne que la faune française. Ces espèces sont les suivantes :

Procladius parvulus K.

Rheopelopia maculipennis (Zett.)

Rh. ornata (Mg.)

Thienemannimyia festiva Fitt.

Zavrelimyia hirtimanus (K.)

Sympotthastia zavreli Pag.

Cricotopus ornatus (Mg.)

C. pilitarsis (Zett.)

C. speciosus $\mathrm{G}$.

Metriocnemus hirticollis (Staeg.)

Pseudosmittia gracilis (G.)

Cryptochiromus rostratus $\mathbf{K}$.

Cryptotendipes nigronitens Edw.

Microtendipes britteni Edw.

Polypedilum quadrigutiatum K.

P. sordens (v. d. W.)
Paratanytarsus bituberculatus (Edw.)

$P$. confusus Pal.

P. laetipes (Zett.)

Tanytarsus usmaensis Pag.

5) Des notes explicatives sont regroupées à la suite de la liste des espèces. Les synonymies données sont limitées aux espèces signalées de France. Des corrections (stations omises, erreurs) et des reports à des opinions d'auteurs récents y sont également indiqués.

6) Dans notre liste on trouvera, en principe, toutes les espèces signalées de France et de Corse, sauf oublis ou omissions de notre part. A côté d'espèces dont le statut est actuellement bien reconnu (mais qui peut éventuellement changer compte tenu des progrès constants accomplis dans la connaissance taxonomique des Chironomidés), nous avons mentionné des espèces décrites il y a fort longtemps notamment par Macquart, par Kieffer ou par Goetghebuer, et qu'on pourrait considérer, vu le laconisme de leurs descriptions et la perte apparente des types, comme nomina dubia. C'est d'ailleurs ce que font Ashe \& Cranston (1990) pour nombre d'entre elles. Or d'autres auteurs, à l'occasion de révisions, comme c'est le cas de Saether (1990) pour le genre Limnophyes, ont été amenés à conserver la validité des noms de certaines de ces espèces. Il nous était difficile, dans ces conditions, de supprimer de notre liste tous les anciens taxa, d'autant plus qu'il n'est pas rare de trouver dans certaines vieilles collections des spécimens provenant manifestement de séries types, comme nous l'avons constaté pour plusieurs Chironomidés décrits par Goetghebuer dont des exemplaires secs appartenant à la série type ont été retrouvés dans les collections Dorier au laboratoire de Zoologie de l'Université de Grenoble. 


\section{Liste des espèces de Chironomidae signalées de France continentale et de Corse jusqu'en 1990}

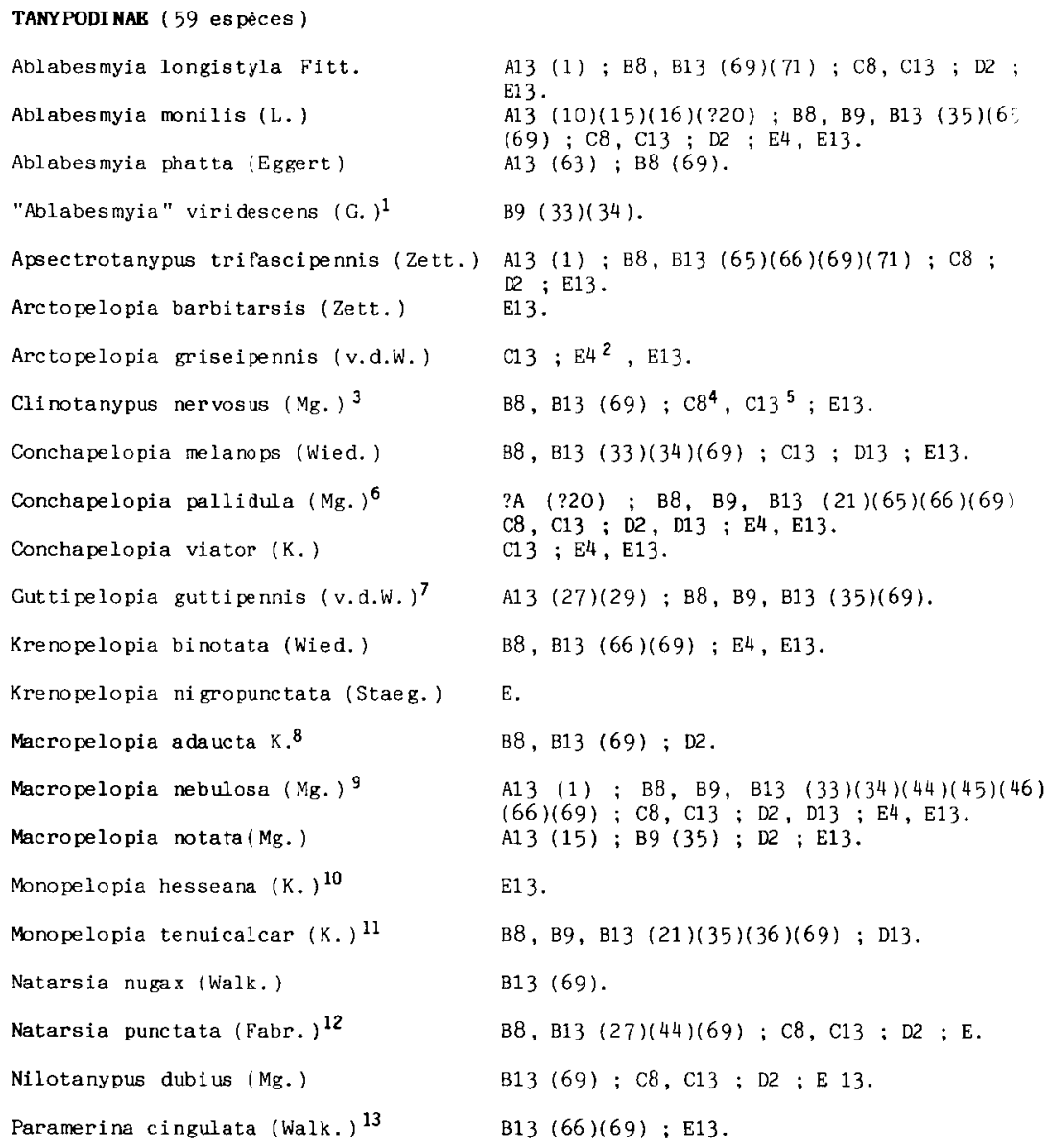


Paramerina divisa (Walk.)

Penta ne urella

katterjokki Fitt. \& Murray

Procladius anomal us K.14

Procladius bifasciatus G, 14

Procladius chore us $(\mathrm{Mg} .)^{16}$

Procladius crassinervis (Zett.)

Procladius culiciformis (L., ${ }^{17}$

Procladius parvulus (K. ) 18

Procladius rivulorum (K.)

Procladius sagittalis (K.)

Procladius signatus (Zett.)

Psectrotanypus varius (Fabr.)

Psilotanypus flavifrons Edw.

Psilotanypus rufovittatus (v.d.W.) 19

Rheopelopia maculipennis (Zett.)

Rheopelopia ornata ( $\mathrm{Mg}$. )

Tanypus ferrugineicollis $\mathrm{Mg} .20$

Tanypus kraatzi (K.)

Tanypus punctipennis $\mathrm{Mg} .21$

Tanypus vilipennis (K.)

Telopelopia fascigera (Verneaux)

Thienemannimyia carnea (Fabr. $)^{23}$

Thienemannimyia gei jskesi (G.)

Thienemannimyia laeta (Mg.)

Thienemannimyia lentiginosa (Fries)
$\mathrm{C} 8 ; \mathrm{D} 2$.

D2,

$B 13(44)$

B9 $(33)(34)^{15}$.

A13 (10)(15)(63) ; B9, B13 (33)(34)(65)(69); $\mathrm{C} 8, \mathrm{C} 13 ; \mathrm{D} 13 ; \mathrm{E} 13$.

?E13.

A13 (27) ; E13.

?E13.

B13 (69).

$\mathrm{B} 8, \mathrm{~B} 13(66)(69) ; ? \mathrm{E} 13$.

C8.

$\mathrm{A} 13(5)(10)(15) ; \mathrm{B} 8, \mathrm{~B} 9, \mathrm{~B} 13(5)(33)(34)(35)$

$(65)(66)(69) ; \mathrm{C} 8, \mathrm{C} 13 ; \mathrm{D} 13 ; \mathrm{E} 13$.

$\mathrm{B} 8, \mathrm{B13}(69)$.

?E13.

B13 (69); PF3 (21).

$\mathrm{B} 13$ (69) ; $\mathrm{C} 13 ; \mathrm{E} 13$.

A13 (1)(5);B9, B13 (5)(35); E13.

A13 (5)(63) ; B8, B13 (5)(69);C8 ; E13.

$\mathrm{B} 8, \mathrm{~B} 9(33)(34)(71) ; \mathrm{E} 4^{22}, \mathrm{E} 13$.

A13 (67) ; B13 (68)(69) ; C13.

$\mathrm{B} 8, \mathrm{~B} 13(44)(69) ; \mathrm{C} 8, \mathrm{C} 13 ; \mathrm{D} 2, \mathrm{D} 13^{24}$.

B13 (66) ; C8 ; D2.

$\mathrm{B} 8, \mathrm{~B} 13$ (69) ; D2, D13 ; E13.

?C8, ?C13 ; ? $13^{25}$ 


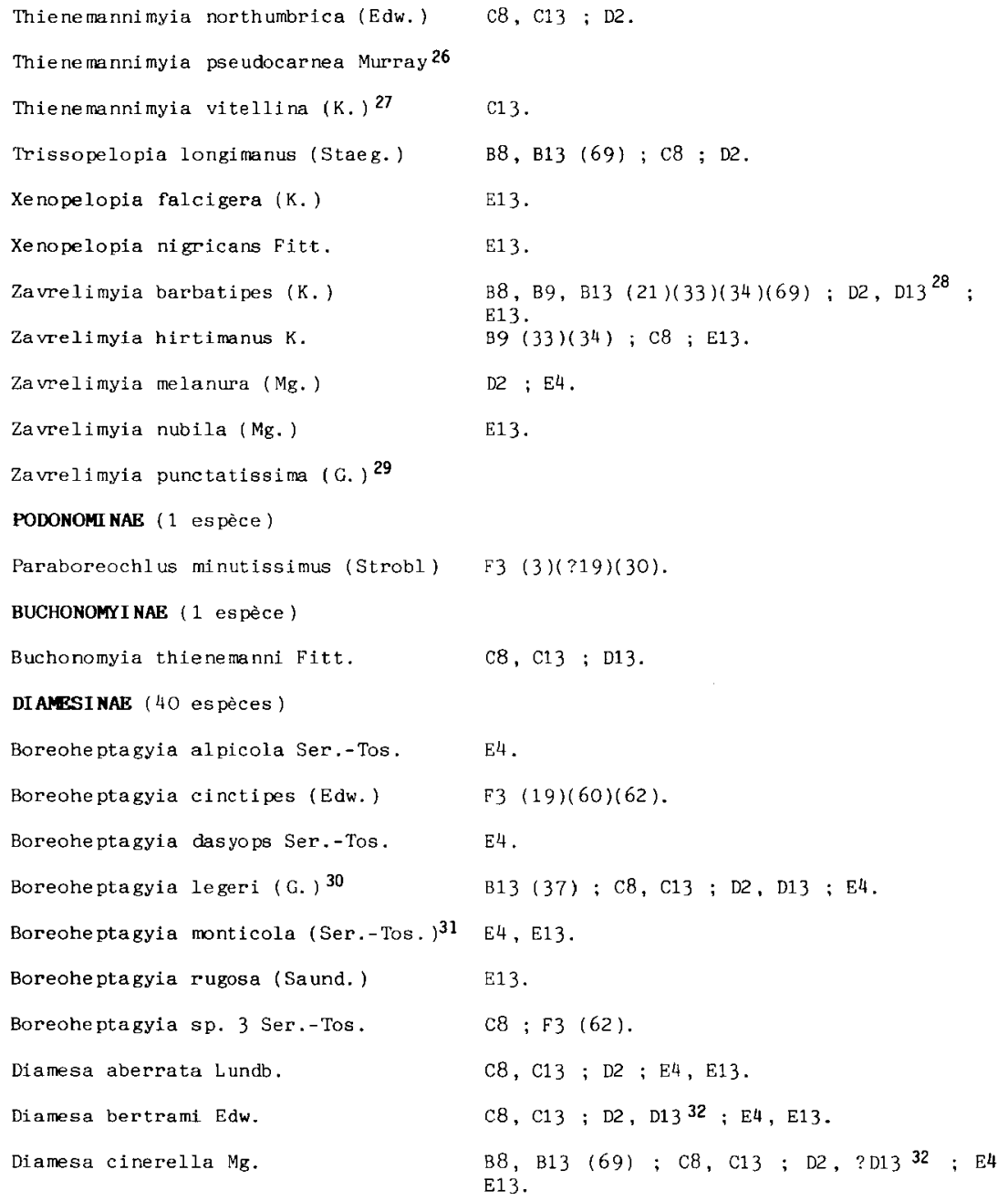


Diamesa confluens K. 33

Diamesa dampfi (K.)

Diamesa goetghebueri Pag. 35

Diamesa hamaticornis $K$.

Diamesa incallida (Walk.)

Diamesa insignipes K.

Dianesa laticauda Ser.-Tos.

Diamesa latitarsis G.

Diamesa lavillei Ser.-Tos.

Diamesa Iindrothi $G$.

Diamesa modesta Ser.-Tos.

Diamesa permacra (Walk.) 37

Diamesa steinboecki G.

Diamesa thomasi Ser.-Tos.

Diamesa tonsa ( $\mathrm{Hal}.)^{38}$

Diamesa vaillanti Ser.-Tos.

Diamesa wuelkeri Ser.-Tos.

Diamesa zernyi Edw.

Onychodiamesa macronyx (K. $)^{42}$

Potthastia gaedii ( $\mathrm{Mg}$.

Potthastia longimanus $\mathrm{K}$.

Potthastia montium $(\mathrm{Edw} .)^{45}$

Pseudodiamesa branickii (Now.) 46

Pseudodiamesa nivosa (G.)

Pseudokiefferiella parva (Edw.)

Sympothastia macrocera Ser.-Tos.
E4 34

E4.

E5.

$\mathrm{C} 8, \mathrm{C} 13 ; \mathrm{D} 2, \mathrm{D} 13^{36}$.

$\mathrm{B} 8(73)(74) ; \mathrm{C} 8, \mathrm{C} 13 ; \mathrm{D} 2 ; \mathrm{E} 4, \mathrm{E} 13$.

$\mathrm{B} 8, \mathrm{~B} 13(69) ; \mathrm{C} 8, \mathrm{C} 13 ; \mathrm{E} 4, \mathrm{E} 13$.

$\mathrm{D} 2 ; \mathrm{E} 4$.

$\mathrm{C} 8 ; \mathrm{D} 2, \mathrm{D} 13^{36} ; \mathrm{E} 4, \mathrm{E} 13 ; ? \mathrm{F3}$ (?19).

D2.

E4.

E4, E.13.

B8 (74); C8; E13.

$\mathrm{D2} ; \mathrm{E} 4$.

D2.

$\mathrm{B} 8, \mathrm{~B} 13(69) ; \mathrm{C} 8, \mathrm{C} 13 ; \mathrm{D} 2 ; \mathrm{E} 4^{39}, \mathrm{E} 13 ;$

?F3 $(? 19)^{40}$.

E4, E13.

$\mathrm{D2} ; \mathrm{E} 4$

$\mathrm{B} 8(74) ; \mathrm{C} 8, \mathrm{C} 13 ; \mathrm{D} 2, \mathrm{D} 13^{41} ; \mathrm{E} 4, \mathrm{E} 13$.

E4 $43 ; \mathrm{F} 3(19)$.

$\mathrm{A} 13(1) ; \mathrm{B} 8, \mathrm{~B} 13(69) ; \mathrm{C} 8, \mathrm{C} 13 ; \mathrm{D} 2, \mathrm{D} 13^{44}$;

E13.

$\mathrm{A} 13$ (1) ; B8, B13 (5)(41)(69); C8, C13 ; D2, D1344; ?E.

$\mathrm{D} 2, \mathrm{D} 13$.

$\mathrm{B} 8(69)(74) ; \mathrm{D} 2 ; \mathrm{E} 4, \mathrm{E} 13$.

$\mathrm{D} 2 ; \mathrm{E} 447, \mathrm{E} 13$.

$\mathrm{B} 8(74) ; \mathrm{D} 2 ; \mathrm{E} 4, \mathrm{E} 13$.

C13. 


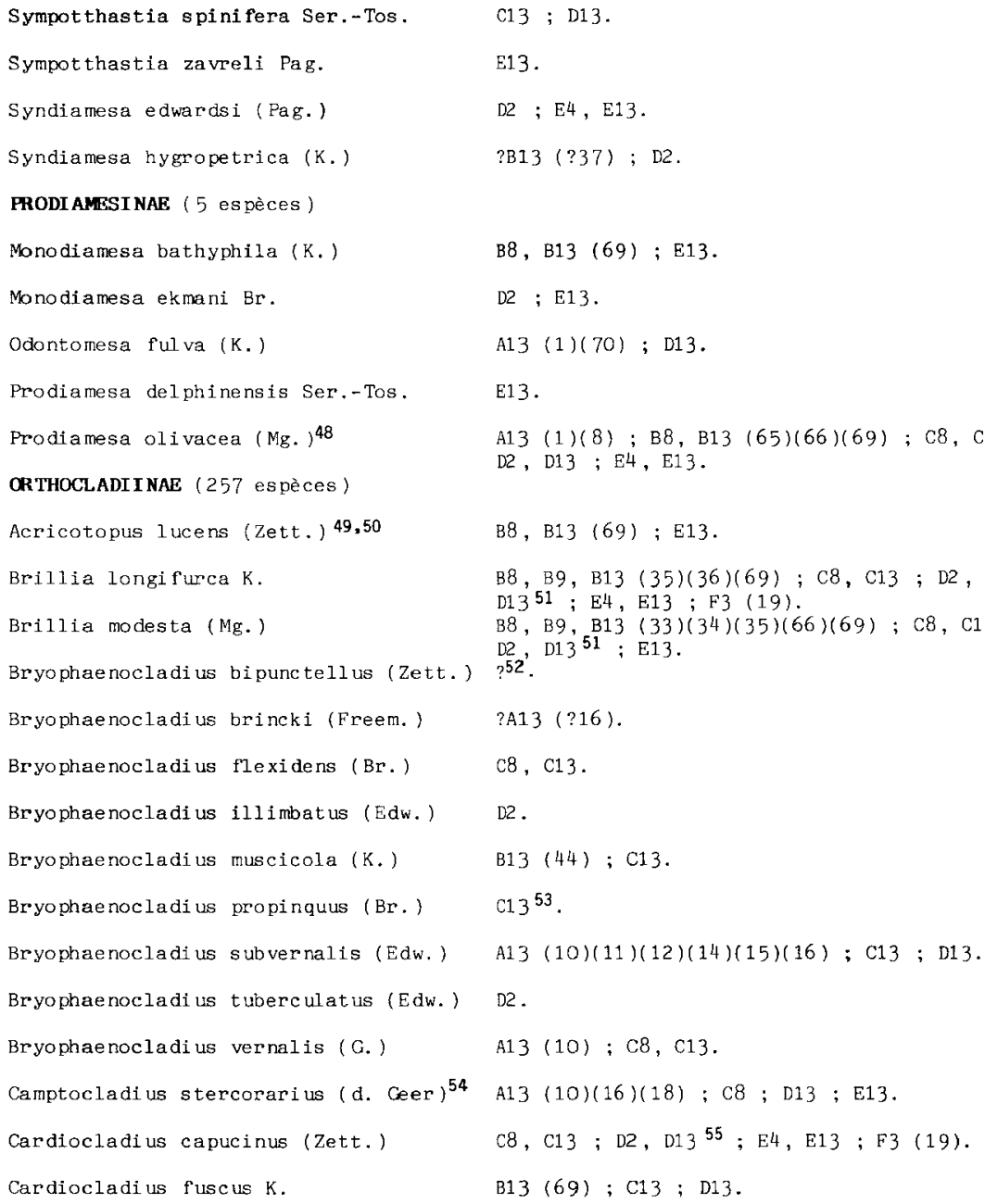


Chaetocladius acuminatus $\mathrm{Br}$.

Chaetocladius acuticornis (K.)

Chaetocladius dentiforceps (Edw.)

Chaetocladius dissipatus (Edw.)

Chaetocladius flavopilosellus G. ${ }^{58}$

Chaetocladius gracilis $\mathrm{Br}$.

Chaetocladius laminatus $\mathrm{Br}$.

Chaetocladius melaleucus ( $\mathrm{Mg}$. )

"Chaetocla di us" mercieri (K.) 60

Chaetocladius perennis ( $M g$. )

Chaetocladius piger (G.)

Chaetocladius suecicus (K.)

Clunio adriaticus Schiner 61

Clunio marinus Halid. 62

Corynoneura bi furcata $K .63$

Corynoneura bitensis K. 64

Corynoneura carriana Edw.

Corynoneura celeripes winn.

Corynoneura celtica Edw.

Corynoneura coronata Edw.

Corynoneure edwardsi Br.

Corynoneura fittkaui Schlee

Corynone ure gratias Schlee 65

Corynone ura kiefferi $G$.

Corynoneura lacustris Edw.

Corynone ura lobata Edw.
E13.

?E13 56 .

A13 (10)(15) : D2.

$C 8^{57} ; E 4$.

B13 (66).

D2 ; E13.

D2 : E4, E13.

$\mathrm{B} 8(39) ; \mathrm{D} 2, \mathrm{D} 13 ; \mathrm{E} 4^{59}$.

A13 (57).

$\mathrm{B} 8, \mathrm{~B} 13(69) ; \mathrm{C} 8, \mathrm{C13} ; \mathrm{D2}, \mathrm{D} 13 ; \mathrm{E} 4, \mathrm{E} 13$.

B9, ?B13 (33)(34)(?69).

D2.

D13 (59).

A13 $(4)(6)(22)(25)(40)(43)(55)(56)(57)$.

E13.

B13 (44).

E13.

B13 (41) ; E13.

B9 (33)(34); ?C8 ; F3 (19).

E13.

C8 ; ?D2 (?23)(?70).

D13.

D2 $2^{66}$.

?A13 (?5) ; C13 ; D2 ; E13.

$\mathrm{C} 8 ; \mathrm{D} 2, \mathrm{D} 13^{67} ; \mathrm{E} 4, \mathrm{E} 13$. 
Corynoneura longistyl us K. ${ }^{68}$

Corynoneura scutellata Winn.

$\mathrm{C} 8, \mathrm{C} 13^{69} ; \mathrm{D} 2 ; \mathrm{E} 13$.

Corynoneura vul garis $K .70$

Cricotopus albiforceps (K.)

?A13 ${ }^{71} ; \mathrm{B} 8(69) ; \mathrm{C} 8, \mathrm{C} 13 ; \mathrm{D} 2^{72} ; \mathrm{E} 13$.

Cricotopus algarum (K.)

$\mathrm{B} 8, \mathrm{~B} 13(69) ; \mathrm{C} 13$; ?E13.

Cricotopus annulator G.

$\mathrm{B} 8, \mathrm{~B} 13(69) ; \mathrm{C} 8, \mathrm{C} 13 ; \mathrm{D} 2, \mathrm{D} 13^{73} ; \mathrm{E} 4$, E13 $75^{2}$ ?F13 $(? 19)^{74}$

Cricatopus beckeri Hirv.

$\mathrm{A} 13^{76} ; \mathrm{B} 8, \mathrm{~B} 9, \mathrm{~B} 13(35)(69) ; \mathrm{C} 8, \mathrm{C} 13 ; \mathrm{D} 2$, E13.

"Cricotopus "coracell us (K.) 78

$\mathrm{B} 13(69) ; \mathrm{C}, \mathrm{C} 13 ; \mathrm{D} 2, \mathrm{D} 13^{79}$.

Cricotopus curtus Hirv.

B9 (35); 013 .

Cricotopus festivellus (K. $)^{80}$

C8, C13; D13; E13.

Cricatopus flavocinctus $\left(\mathrm{K}, 1^{81}\right.$

$\mathrm{B} 8, \mathrm{~B} 13(66)^{82}(69) ; \mathrm{D} 2 ; \mathrm{E} 13$.

Cricatopus fuscus (K.)

$\mathrm{C} 13^{83}, \mathrm{E} 13$.

Cricotopus laricomalis Edw.

C13 ; D2 ; E13.

Cricotopus lygropis Edw.

$\mathrm{C} 13 ; \mathrm{E} 13^{85}$.

Cricotopus ornatus (Mg. $)^{84}$

E13.

Cricatopus pallidipes Edw.

B8 (69); C13.

Cricotopus pilitarsis (Zett.)

D2 ; E13.

Cricotopus pirifer Hirv.

B13 (44).

Cricotopus polychromus K. ${ }^{86}$

?B8 (?32) ; $\mathrm{C8} ; \mathrm{E4}$.

Cricotopus pulchripes Verr. ${ }^{87}$

$\mathrm{B} 8, \mathrm{~B} 13$ (69); $\mathrm{C} 8, \mathrm{C} 13 ; \mathrm{D} 2, \mathrm{D} 13^{88}$.

Cricotopus similis G.

$\mathrm{B} 8, \mathrm{~B} 13(69) ; \mathrm{C} 13$.

Cricotopus speciosus G.

B8 (69).

Cricotopus suspiciosus Hirv.

A13 (1)(5)(?53) ; B8, B9, B13 (33)(34)(35) $(? 65)(69)(71) ; \mathrm{C} 8, \mathrm{C} 13 ; \mathrm{D} 2 ; \mathrm{E} 13$.

?A13 (?51)(?54) ; B8, B13 (35)(36)(63)(69) ; $\mathrm{C} 13^{90}$, E13. 
Cricotopus tremul us (L.) 91

Cricotopus triannulatus (Macq.)

Cricotopus tricinctus ( $\mathrm{Mg}$. )

Cricotopus trifascia Edw.

Cricotopus trifasciatus ( $\mathrm{Mg}.)^{93}$

Cricotopus vierriensis G. 94

Diplocladius cultriger K.

Epoicocladi us flavens $(\operatorname{Mall} \text {. })^{96}$

Eukiefferiella alpestris G.

Eukiefferiella brevicalcar (K.)

Eukiefferiella claripennis (Lundb,) 97

Eukiefferiella clypeata (K.)

Eukiefferiella coerulescens (K.)

Eukiefferiella cyanea Th.

Eukiefferiella devonica (Edw.)

Eukiefferiella dittmari Lehm.

Eukiefferiella fittkaui Lehm.

Eukie f f'eriella ful densis Lehm.

"Eukiefferiella" fusicornis G. ${ }^{99}$

Eukiefferiella gracei (Edw.) 100

Eukiefferiella ilkleyensis (Edw.)

Eukiefferiella lobifera G.

Eukiefferiella minor (Edw.) 101

Eukiefferiella pseudomontana $G$.

Eukiefferiella similis G.

Eukiefferiella tirolensis G.
A13 (53)(54) ; B8, B13 (69); C8, C13 ; D2, D13 92 ; E13.

A13 (53)(54);B8, B13 (66)(69); C8, C13 ;

D13 ; E13.

A13 (53)(54); C8, C13 ; D2 ; E13.

$\mathrm{B} 8, \mathrm{~B} 13$ (69); C8, C13; D13 ; E13.

A13 (1) ; B8, B13 (69) ; C8, C13 ; D2 ; E13.

B13 (69); C13 ; ?D1395 ; E13.

$\mathrm{B} 8, \mathrm{~B} 13(66)(69) ; \mathrm{C} 13 ; \mathrm{D} 13 ; \mathrm{E} 13$.

B8, B13 (69) ; D13.

E4.

$\mathrm{B} 8, \mathrm{~B} 13$ (69); C8, C13; D13; E13.

A13 (1); B8, B9, B13 (35)(66)(69); C8, C13;

$\mathrm{D} 2, \mathrm{D} 1398 ; \mathrm{E} 4, \mathrm{E} 13$.

$\mathrm{C} 8, \mathrm{C} 13$; D2, 13 .

$\mathrm{C} 8, \mathrm{C} 13 ; \mathrm{D} 2, \mathrm{D} 13 ; \mathrm{E} 4, \mathrm{E} 13$.

D2, $\mathrm{V} 13^{98}$.

$\mathrm{A} 13$ (1) ; C8, C13 ; D2, D13 ; E13.

C8, C13 ; ?E13.

D2, D13 ; E4, E13.

$\mathrm{C} 8, \mathrm{C} 13 ; \mathrm{D} 2, \mathrm{D} 1398$.

B13(33)(34).

C8, C13; D13.

A13 (1) ; C8, C13 ; D13.

C8, C13 ; D13 ; E13.

$\mathrm{B} 8, \mathrm{~B} 13$ (69); $\mathrm{C} 13 ; \mathrm{D} 2, \mathrm{D} 13^{102} ; \mathrm{E} 4, \mathrm{E} 13$.

C8 ; D13 ; E13.

$\mathrm{C} 8, \mathrm{C} 13 ; \mathrm{D} 2, \mathrm{D} 13$.

$\mathrm{C} 13 ; \mathrm{D} 2, \mathrm{D} 13 ; \mathrm{E} 4, \mathrm{E} 13$. 
Eurycnemus crassipes (Panz.) 103

Gymnometriocnemus brumal is (Edw.)

Halocladi us braunsi (G.)

Halocladius fucicola (Edw.)

Halocladius millenarius (Sant. A.) 105

Halocladi us variabilis (Staeg.) 106

Halocladius varians (Staeg.) 109

Heleniella dorieri Ser.-Tos.

Heleniella ornaticolis (Edw. ) ${ }^{111}$

Heleniella serratosioi Ringe

Heterotanytarsus apicalis (K.)

Heterotrissocladius marcidus (Walk.)

Hydrobaenus distylus (K.)

Hydrobaenus lugubris Fries

Hydrobaenus spinatis Saeth.

Krenosmittia boreoalpina G.

Kronosmittia camptophleps (Edw.)

Limnophyes acutus G. ${ }^{115}$

Limnophyes as quamatus And.116

Limnophyes difficilis $\mathrm{Br}$.

Limnophyes foliatus (K.) 118

Limnophyes gurgicola Edw.

Limnophyes habilis (Walk.) 119

Limnophyes minimus (Mg. $)^{120,121}$

Limnophyes natalensis (K.) 124

Limnophyes pentaplastus $\left(K_{.}\right)^{126}$
A13 (28)(31).

A13 (10)(15)(16) ; D2 ; E4, E13.

E13.

D13 $104 ; \mathrm{E} 13$.

E13.

A13 (2) 107 ; ?E13 108 .

$\mathrm{C} 13110 ; \mathrm{E} 13$.

E4.

$\mathrm{C} 8, \mathrm{C} 13 ; \mathrm{D} 2, \mathrm{D} 13^{112} ; \mathrm{E} 13$.

E13.

D2.

$\mathrm{B} 8, \mathrm{~B} 13(66)(69) ; \mathrm{C} 13 ; \mathrm{D} 2 ; \mathrm{E} 4^{113}$.

E4.

A13 (26).

E4.

D2 ; E4.

$\mathrm{C} 8, \mathrm{C} 13 ; \mathrm{D2}, \mathrm{D} 13^{114}$.

?B8, ?B13 (?69).

D2 ; E4.

$\mathrm{C13}$; ? D13 117.

B13 (44).

$\mathrm{C} 8, \mathrm{C} 13 ; \mathrm{D} 2 ; \mathrm{E} 4$.

$\mathrm{B} 8, \mathrm{~B} 13$ (69) ; $\mathrm{C} 13 ; 02$.

A13 $(10)(11)(12)(14)(15)(16) ; B 8, B 13(69) ; C 8$, $\mathrm{C} 13^{122}, \mathrm{D} 2 ; \mathrm{E} 13 ; ? \mathrm{~F} 3(? 19)^{123}(? 28)^{123}(? 31)^{123}$. A13 (16) ; ?D 125 .

$\mathrm{B} 8, \mathrm{~B} 13(47)(66)(69) ; \mathrm{C} 8, \mathrm{C} 13 ; \mathrm{D} 2, \mathrm{D} 13^{127}$; E4, E13. 


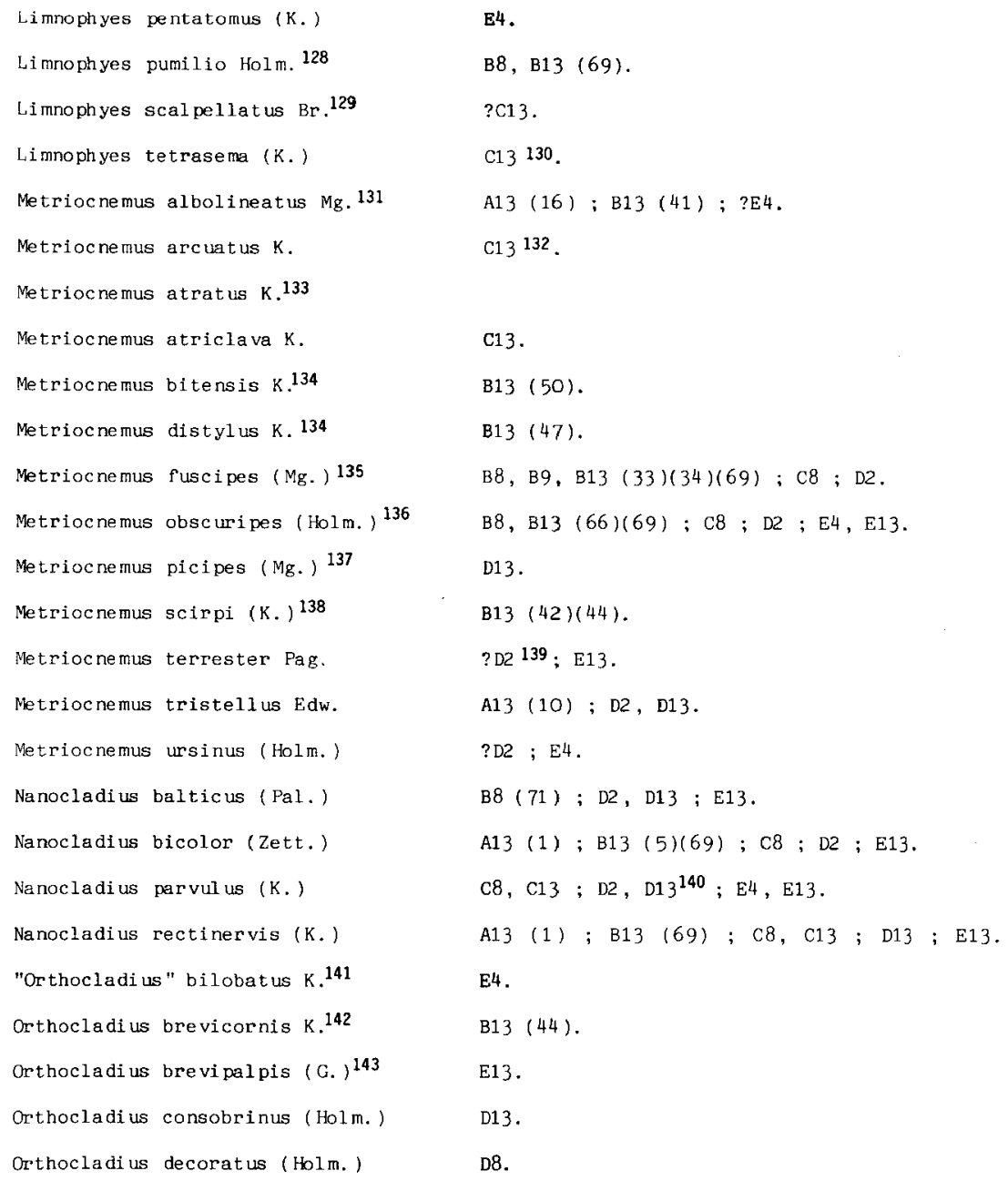


Orthocladius dentifer Br.

Orthocladius excavatus $\mathrm{Br}$.

Orthocladius frigidus (Zett.)

Orthocladius fuscimanus (K.)

Orthocladius fusiformis G. 145

Orthocladius glabripennis (G.)

Orthocladius kiefferiellus G. 146

Orthocladius lignicola K.

Orthocladius lobulifer G. 147

Orthocladius meridionalis G. 147

Orthocladius mixtus (Holm.)

Orthocladius oblidens (Walk.)

Orthocladius obtexens $\mathrm{Br} .{ }^{148}$

Orthocladius rhyacobius $k$.

Orthocladius rivicola $\mathrm{K}$.

Orthocladius rivulorum $K$.

Or thocladius rubicundus ( $\mathrm{Mg}$. )

Orthocladius saxicola $K$.

Orthocladius saxosus (Tok.)

Orthocladius smolandicus $\mathrm{Br}$.

Orthocladius thienemanni $K$.

Orthocladius timoni C. 150

Orthocladius wetterensis $\mathrm{Br}$.

Paracladius alpicola (Zett.)

Paracladius conversus (Walk.)

Paracladius quadrinodos us Hirv.
D8.

A13 (1) ; ?B (?69) ; C8, G13 ; D2, D13 ; E13.

A13 (1) ; B8, B13 (69); C8, C13; D2, D13 : E4, E13.

$\mathrm{CB}, \mathrm{C} 13 ; \mathrm{D} 2, \mathrm{D} 13^{144} ; \mathrm{E}^{4}, \mathrm{E} 13$.

E13.

c13.

B13 (44).

B13 (66)(?69);C8.

E13.

E13.

E4.

?B (?69);C8, C13 ; D2 ; E13.

$\mathrm{B} 8, \mathrm{~B} 13(69) ; \mathrm{C} 8, \mathrm{C} 13 ; \mathrm{E} 4, \mathrm{E} 13$.

D2.

$\mathrm{B} 8$ (39) ; $\mathrm{C} 8, \mathrm{C} 13 ; \mathrm{D} 2, \mathrm{D} 13 ; \mathrm{E} 4, \mathrm{E} 13$.

?B8 (?69); $\mathrm{C} 8, \mathrm{C13} ; \mathrm{D} 2, \mathrm{D} 13^{149} ; \mathrm{E} 4, \mathrm{E} 13$.

A13 (1) ; B8, B9 (35)(66); C8, C13 ; D2 ; E4, E13.

$\mathrm{A} 13(1) ; ? \mathrm{~B}($ ?69) ; C8, C13 ; D2, D13.

C8 : D2, D13 ${ }^{149}$.

D2.

$\mathrm{B} 8, \mathrm{~B} 13(69) ; \mathrm{C} 8, \mathrm{C} 13 ; \mathrm{D} 2, \mathrm{D} 13 ; \mathrm{E} 13$.

E13.

E4, E13.

D2 ; E4 ${ }^{151}, \mathrm{E} 13$.

A13 (1) ; B13 (66) $)^{152}(69) ; \mathrm{C} 8, \mathrm{Cl3} ; \mathrm{D} 13$; E13.

B13 (69). 


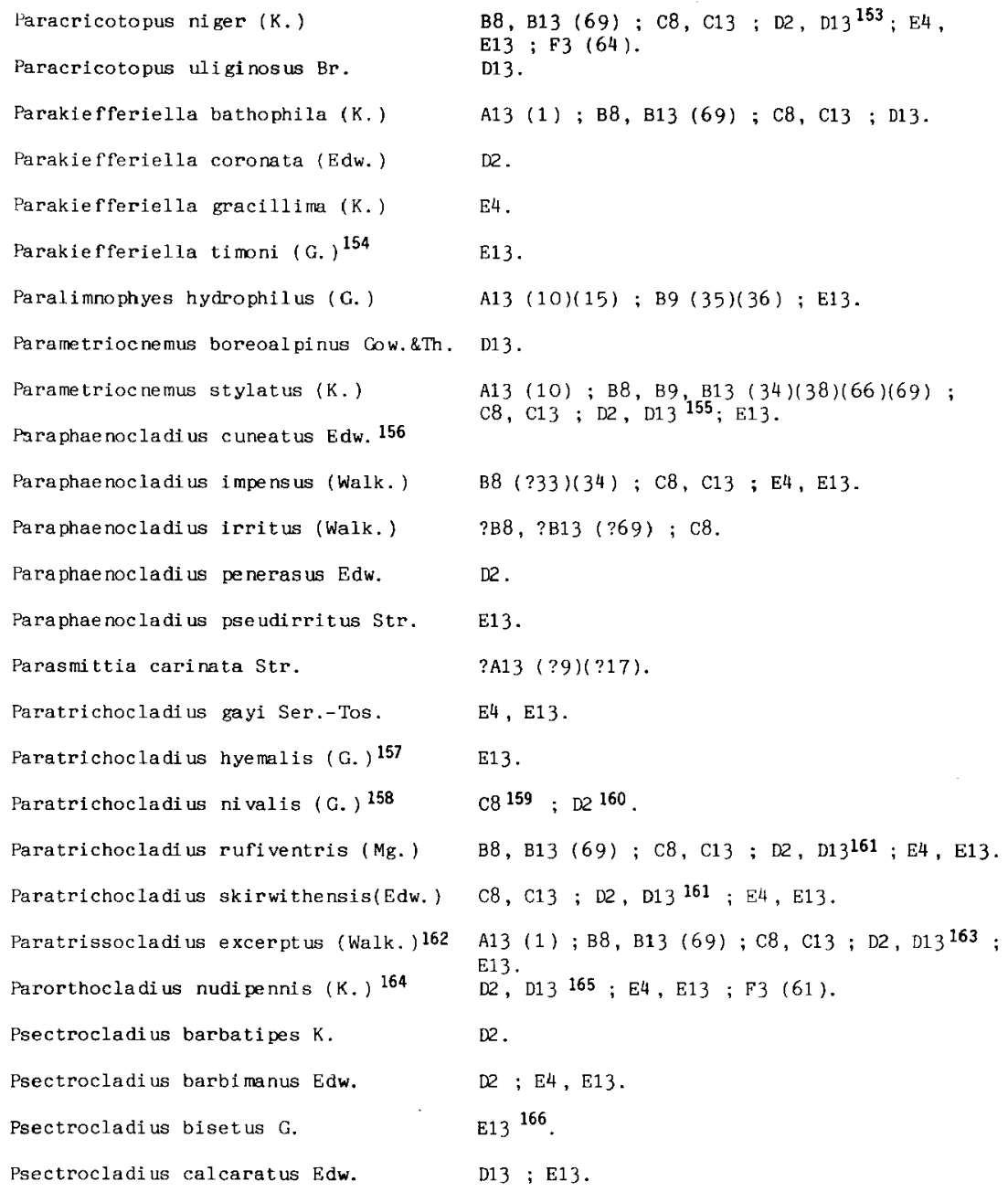




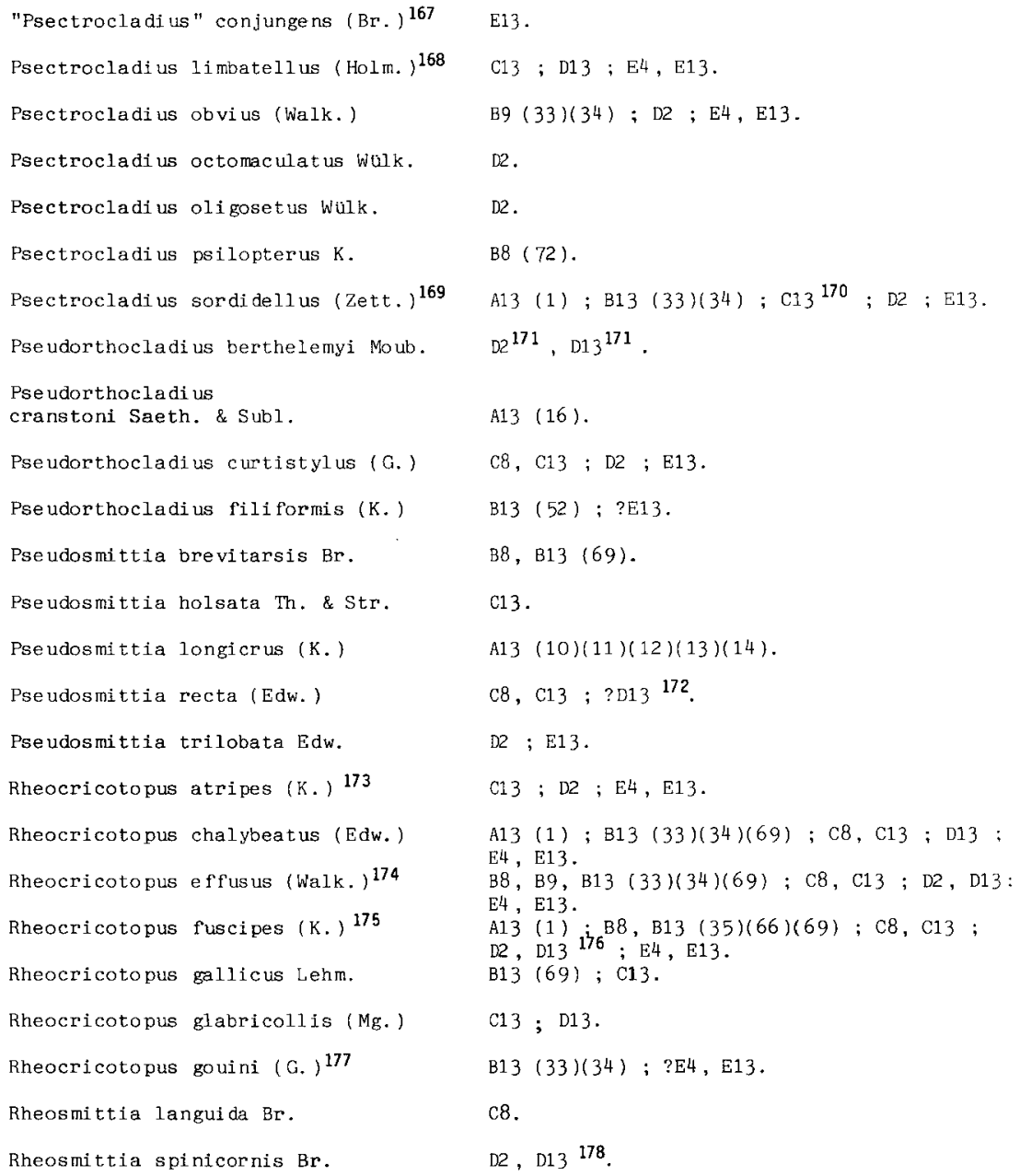


Smittia alpicola $G$.

Smittia alpina (K.) 179

Smittia aterrima (Mg.) 180

"Smittia" bicolorata (G.) 181

Smittia davidi G. 182

Smittia lacroixi (K.)

Smittia leucopogon (Mg.) 184

Smittia longirostris G. 185

Smittia nudipennis (G.)

Smittia pratorum $G$.

Smittia rupicola (K.)

Smittia superata G. 187

Smittia thalassicola G. 189

Smittia vesparum (G. ) 190

Symbiocladius rhithrogenae (Zav.) 191

Synorthocladius semivitrens (K. ) 193

Thalassosmittia

thalassophila (Beq. \& G.) 195

Thienemannia gracei (Edw.)

Thienemannia gracilis K.196

Thienemannia libanica Lav. \& Moub .

Thienemanniella acuticornis (K.) 197

Thienemanniella clavicornis $\mathrm{K}$.

Thienemanniella emarginata K.198

Thienemaniella flavescens Edw.

Thienemanniella lutea Edw.
E13.

E.

$\mathrm{A} 13(16) ; \mathrm{B} 8, \mathrm{~B} 13(? 44)(69) ; \mathrm{C} 8, \mathrm{C13} ; \mathrm{D} 2$; E13.

E4.

E13.

C13 183 .

D13.

E4.

$\mathrm{C} 8, \mathrm{C} 13^{186} ; \mathrm{D} 13$.

A13 (10)(11)(13)(14)(15);C8, C13;D13;

E13.

A13 (57).

C13 188 .

E13.

B8 (39) ; D13192;E13.

$\mathrm{A} 13$ (1) ; B8, B13 (69); C8, C13; D2, D13 194 ; E4, E13.

C8; $; 13 ; E 4$.

$\mathrm{B} 8, \mathrm{~B} 13(66)(69) ; \mathrm{D} 13 ; \mathrm{E} 13$.

E4.

C8 ; D13 ; E13.

$\mathrm{B} 13$ (63); $\mathrm{C} 8 ; \mathrm{D} 2 ; \mathrm{E} 4, \mathrm{E} 13$.

B13(51).

D13.

D13. 
Thienemanniella majuscula (Edw.)

Thienemanniella obscura $\mathrm{Br}$.

Thienemanniella partita Schlee

Thienemanniella vittata Edw.

Tokunagaia rectangularis (G.)

"Trichocladi us" ampul laceus K. 200

Tvetenia bavarica (G.)

Tvetenia calvescens (Edw.)

Tvetenia discoloripes (G.)

Tvetenia veralli (Edw.)

CHIRONOMI NaF CHIRONOMI NI (142 espèces)

Camptochironomus

pallidivittatus (Mall.)

Camptochironomus tentans (Fabr.)

Chironomus annularius

auct. nec de Ceer 202. 203

Chironomus annulatus Macq. 204

Chironomus anthracinus Zett.

Chironomus aprilinus Mg. 205, 206

Chironomus bernensis klotzli

Chironomus calipterus $\mathrm{K},{ }^{208}$

Chironomus cingulatus ( $\mathrm{Mg}.)^{209}$

Chironomus commutatus Str.

Chironomus dorsalis auct. nec $\mathrm{Mg} .{ }^{210}$

Chironomus gracilis Macq. 211

Chironomus humeralis Macq. 211

Chironomus longistylus $\mathrm{C}$.
$013^{199}$.

$\mathrm{C} 8, \mathrm{C} 13 ; \mathrm{D} 13 ; \mathrm{E} 13$.

?C13.

$\mathrm{C} 8, \mathrm{C} 13 ; \mathrm{D} 2, \mathrm{D} 13^{199}$.

E4.

B13 ( 52$)$.

$\mathrm{C} 8 ; \mathrm{D2}, \mathrm{D} 13^{201} ; \mathrm{E} 4, \mathrm{E} 13$.

$\mathrm{A} 13$ (1) ; $\mathrm{B} 8, \mathrm{~B} 13(69) ; \mathrm{C} 8, \mathrm{C} 13 ; \mathrm{D} 2, \mathrm{D} 13$ :

E4, E13.

C13 ; D13.

$\mathrm{C} 8, \mathrm{C} 13 ; \mathrm{D} 2, \mathrm{D} 13^{201} ; \mathrm{E} 4, \mathrm{E} 13$.

$\mathrm{B} 8$ (69); C8 ; E13.

B13 (41) ; E13.

?A13 (?5) ; B13 (69); C8, C13 ; E13.

A13 (53).

B8 (69)(?71) ; C13 ; D2 ; E13.

E13.

$\mathrm{C13} ;$ ? $13^{207}$.

E13.

?A13 (?53) ; B8, B9, B13 (35)(69); C13 ; D13 : E13. D2 ; E13.

$\mathrm{A} 13(54) ; \mathrm{B} 8, \mathrm{~B} 9, \mathrm{B13}(33)(34)(35)(65)(69)$; $\mathrm{C} 8, \mathrm{C} 13 ; \mathrm{E} 13$.

A13 (53).

A13 (53).

E13. 


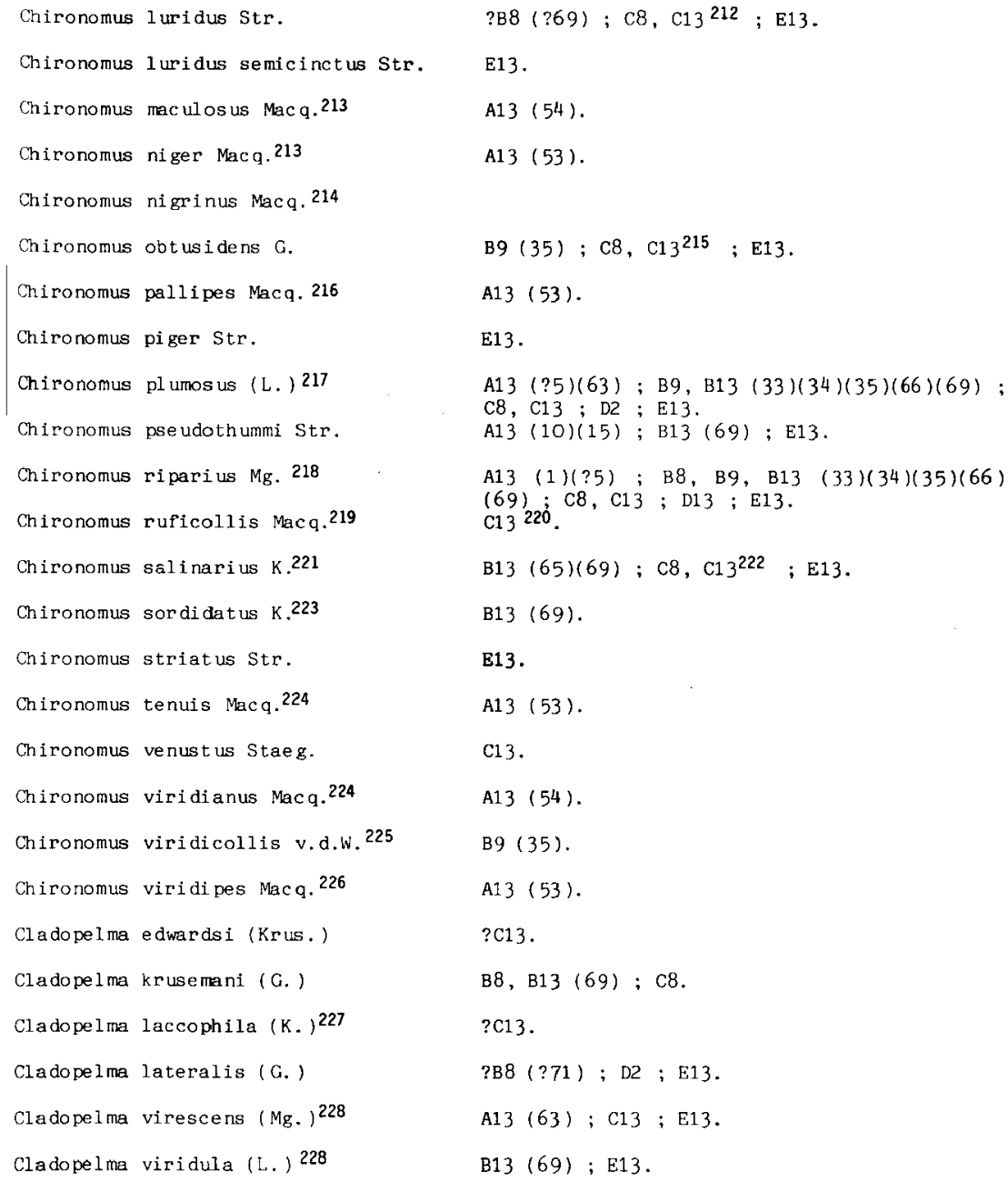




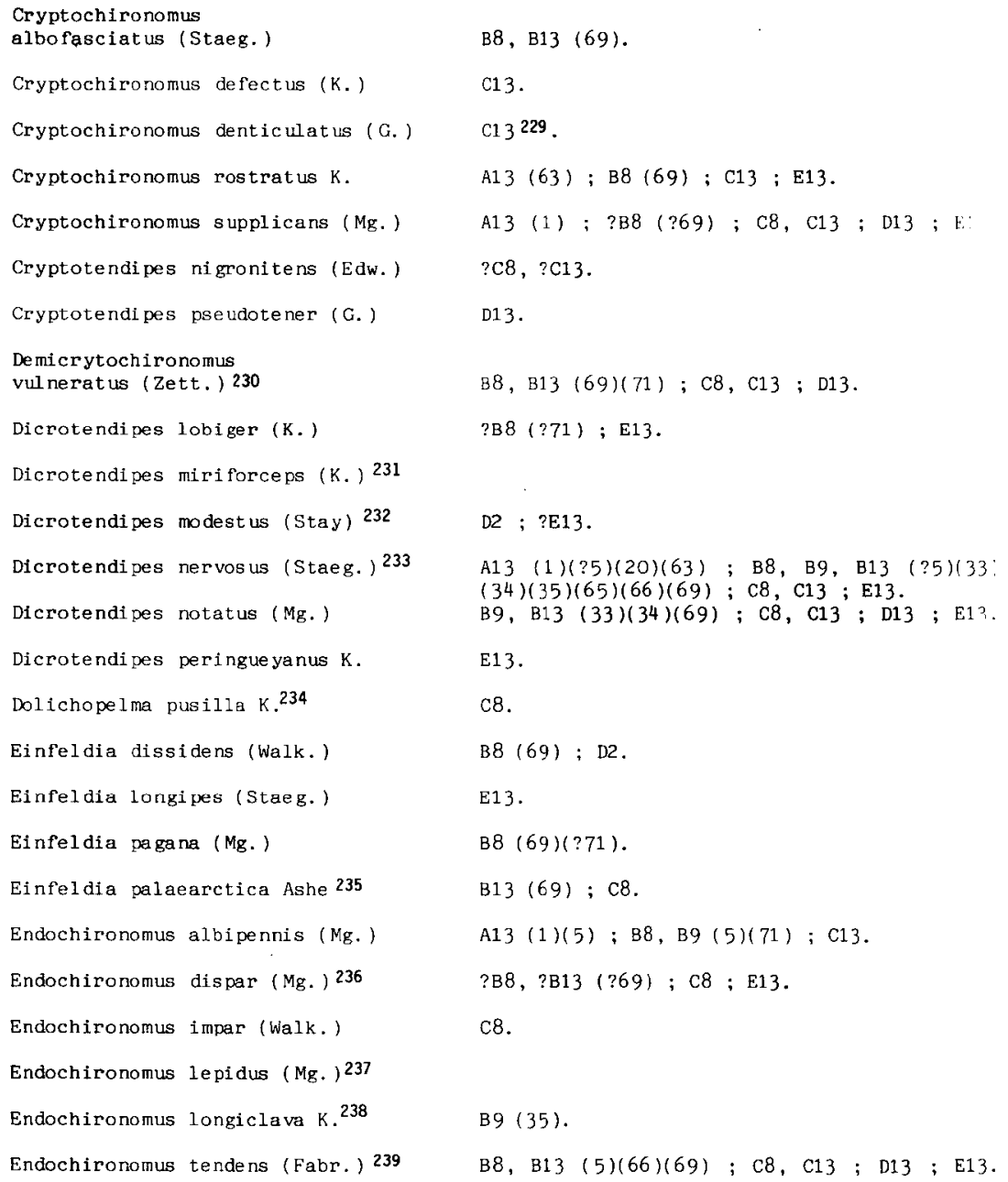


yptotendi pes barbipes (Staeg.)

Glyptotendipes foliicola $\mathrm{K} .{ }^{240}$

Gl yptotendi pes fulvofasciatus $K$.

Glyptotendi pes glaucus ( $M g$. )

Glyptotendipes gripekoveni (K.)

Glyptotendi pes pallens ( $\mathrm{Mg}$.)

Glyptotendipes paripes Edw.

Glyptotendipes rufipes (L.) 242

Glyptotendi pes sigillatus K. 243

Glyptotendipes viridis (Macq.) 244

Harnischia curtilamellata (Mall.) 245

Harnischia fuscimanus $K$.

Kiefferul us tendipediformis (G.)

Lauterborniella agrayloides (K.)

Laterborniella fuscoguttata (K.)

Microchironomus deribae (Freem, ${ }^{246}$

Microchironomus tener (K.)

Microtendipes britteni Edw.

Microtendipes chloris ( Mg.) 247

Microtendipes confinis (Mg.)

Microtendipes diffinis Edw.

Microtendipes nitidus (Mg. $)^{247}$

Microtendipes pedellus (de Ceer) 247

Microtendipes rydalensis (Edw.)

Omis us caledonicus (Edw.)

Pagastiella orophila (Edw.)
B9 (33)(34);E13.

$\mathrm{C} 8, \mathrm{C} 13^{241}$.

B13 (69).

B9 $(33)(34)(35)$.

?A13 (?5)(?20) ; B8, B13 (?5)(65)(66)(69);

C8 ; D13 ; E13.

A13 (1)(35);B13 (35); C13.

A13 (1) ; B9 (33)(34);C13.

A13 (48).

B13 (69); C13;E13.

C13 ; E13.

B8, B13 (66)(69);C13 ; E13.

E13.

E13.

C13;E13.

A13 (5)(63);B13 (69); C13 ; E13.

B8, B13 (69).

?B8 (?71) ; $\mathrm{C} 8, \mathrm{C} 13$.

C8 ; D13.

E13.

$\mathrm{B} 8, \mathrm{~B} 9, \mathrm{~B} 13(? 5)(33)(34)(35)(69) ; \mathrm{C} 8, \mathrm{C} 13$; D2 ; E13.

$\mathrm{C} 8248, \mathrm{C13} ; \mathrm{D} 13$.

c8.

B8 (69)(71); D2. 


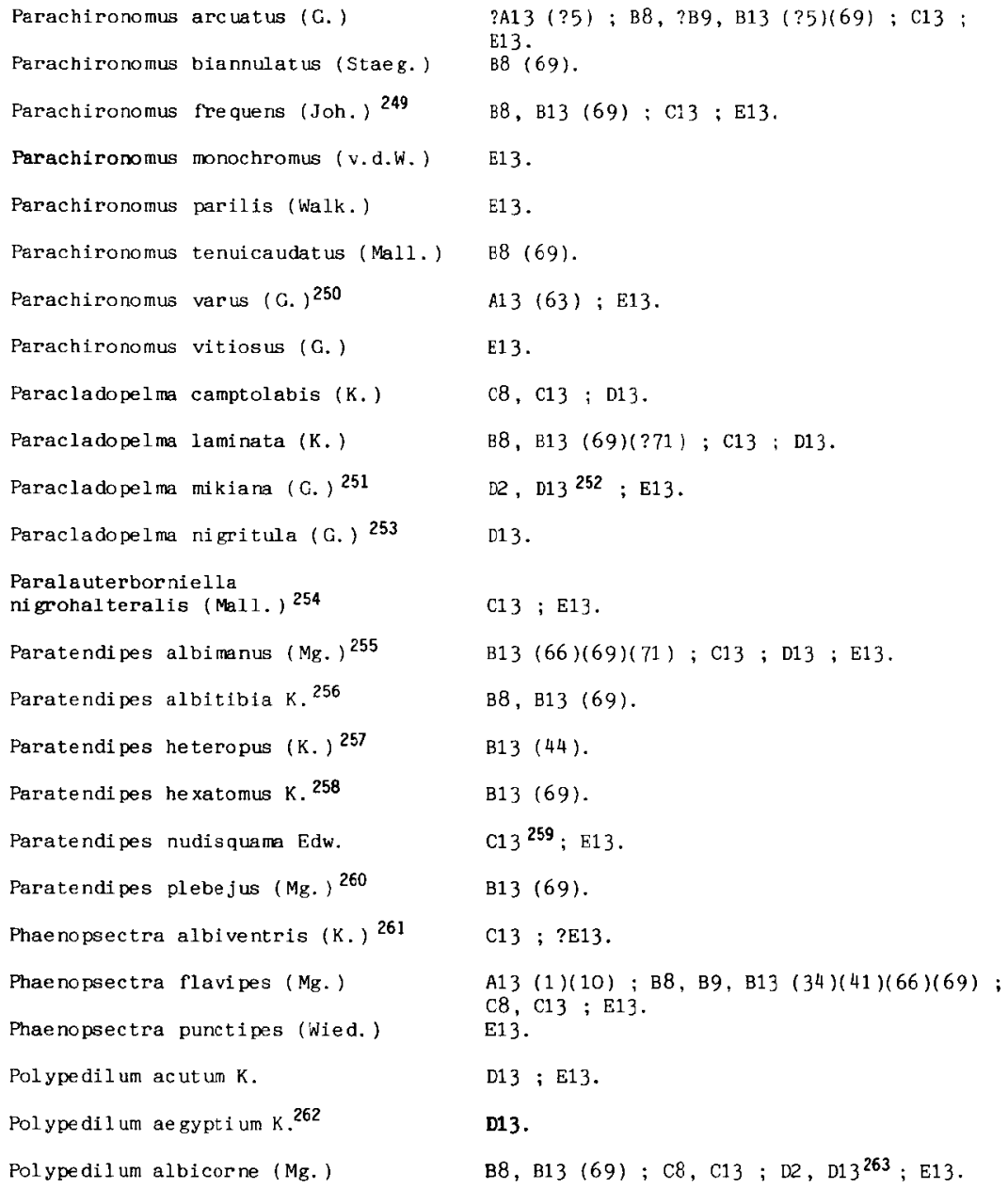




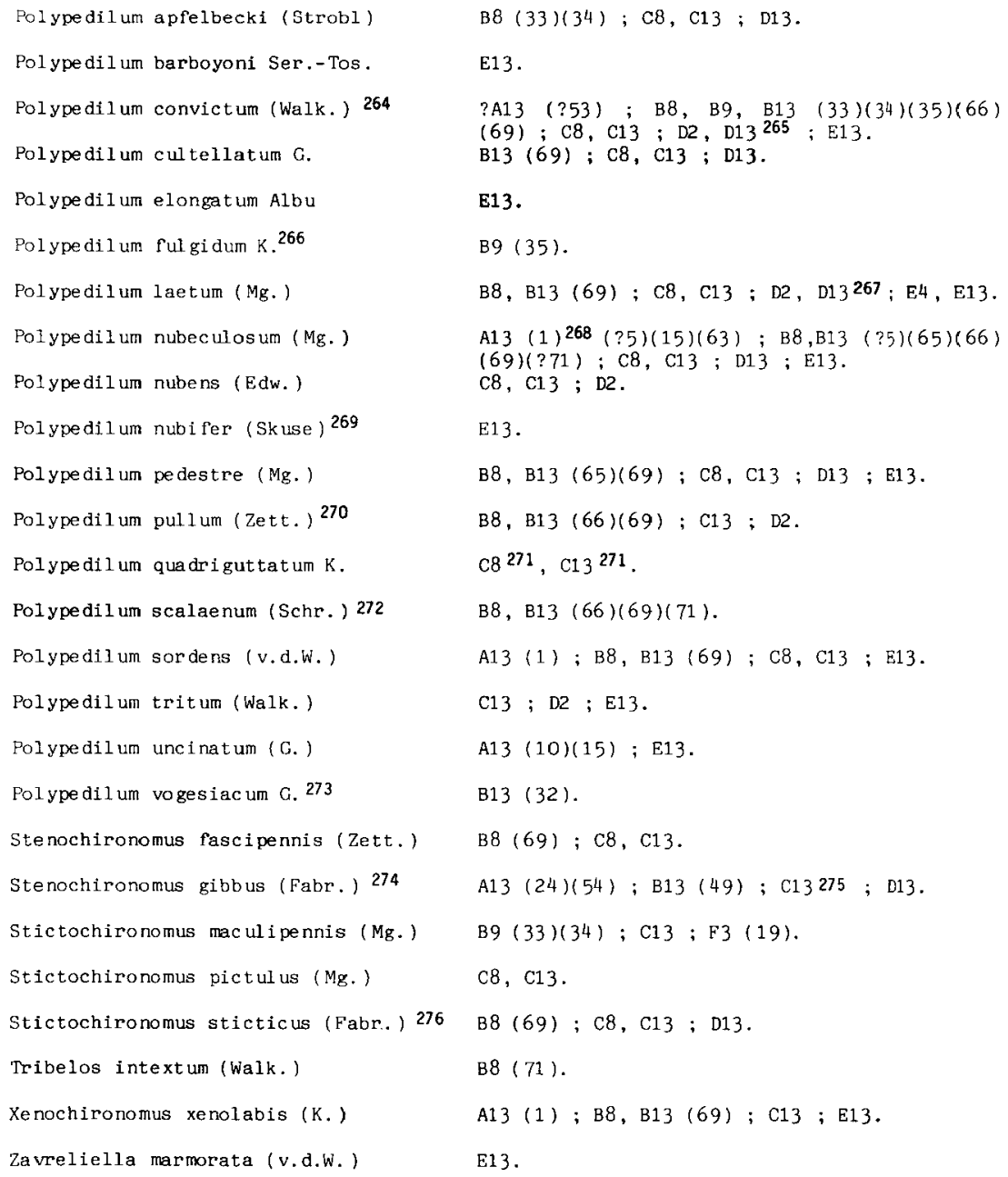




\section{CHIRONOMI NAE PSEUDOCHI RONOMI NI (1 es pèce )}

Pseudochironomus prasinatus (Staeg.) B8, B13 (69).

CHIRONOMI NAB TANYTARSINI ( 90 espèces)

Cladotanytarsus atridorsum $K$.

Cladotanytarsus i ucundus Hirv.

Cladotanytarsus mancus (Walk.)

Cladotanytarsus vanderwulpi (Edw.)

"Cowiniella bavarica" Thien. 277

Krenopsectra fallax Reiss

Lithotanytarsus emarginatus (C.)

Micropsectra apposita (Walk.)

Micropsectra atrofasciata (K.)

Micropsectra auvergnensis Reiss

Micropsectra bi dentata G.

"Micropsectra" chlorophila (K. $)^{280}$

Micropsectra clastrieri Reiss

Micropsectra contracta Reiss

Micropsectra fusca (Mg.)

Micropsectra globulifera (G. $)^{281}$

Micropsectra heptameris K. 281

Micropsectra junci ( Mg. ) 283

Micropsectra Iindrothi G. 284

Micropsectra notescens (Walk.) 286

Micropsectra radialis G. 287

Micropsectra recurvata G.

Neostempellina thienemanni Reiss
$\mathrm{B} 8, \mathrm{~B} 13(69) ; \mathrm{C} 8, \mathrm{C} 13 ; \mathrm{D} 2 ; \mathrm{E} 13$.

02.

$\mathrm{A} 13$ (63) ; $\mathrm{B} 8, \mathrm{~B} 13(69) ; \mathrm{C} 8, \mathrm{C} 13 ; \mathrm{D} 1 \%$ E13.

C13.

$\mathrm{C} 8{ }^{278} ; \mathrm{E} 13$.

02.

D2.

D2.

$\mathrm{A} 13(1) ; \mathrm{B} 8, \mathrm{~B} 13(65)(69) ; \mathrm{C} 8, \mathrm{C} 13 ; \mathrm{D} 2$, $\mathrm{D} 13 ; \mathrm{E} 4, \mathrm{E} 13$. C 279 .

$\mathrm{B} 8, \mathrm{~B} 13(65)(69) ; \mathrm{C} 8 ; \mathrm{D} 2, \mathrm{D} 13 ; \mathrm{E} 4, \mathrm{E} 13$.

B13 (48).

54.

$\mathrm{D2}$.

$\mathrm{A} 13(10)(15) ; \mathrm{B} 8, \mathrm{~B} 13(69) ; \mathrm{C} 8 ; \mathrm{D} 2 ; \mathrm{E} 1$

C8.

$\mathrm{E} 4282$

$\mathrm{C} 8 ; \mathrm{D} 2 ; \mathrm{E} 13$.

$\mathrm{D} 2, ? \mathrm{D} 13^{285} ; \mathrm{E} 13$.

$\mathrm{B} 8, \mathrm{~B} 13(66)(69) ; \mathrm{C} 8, \mathrm{C} 13 ; \mathrm{D} 2 ; \mathrm{E} 4, \mathrm{E} 13$.

D2.

D2.

E13. 


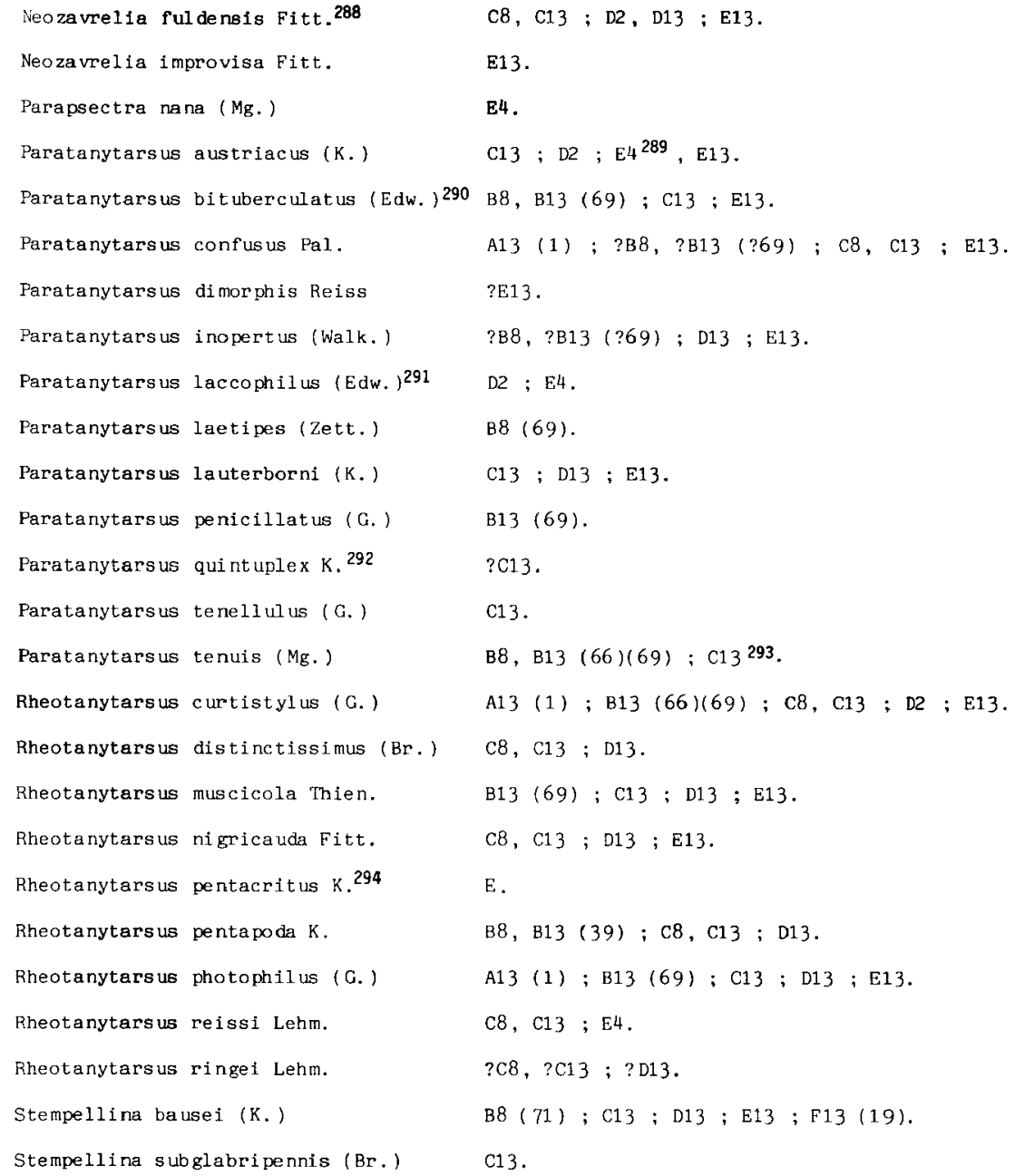




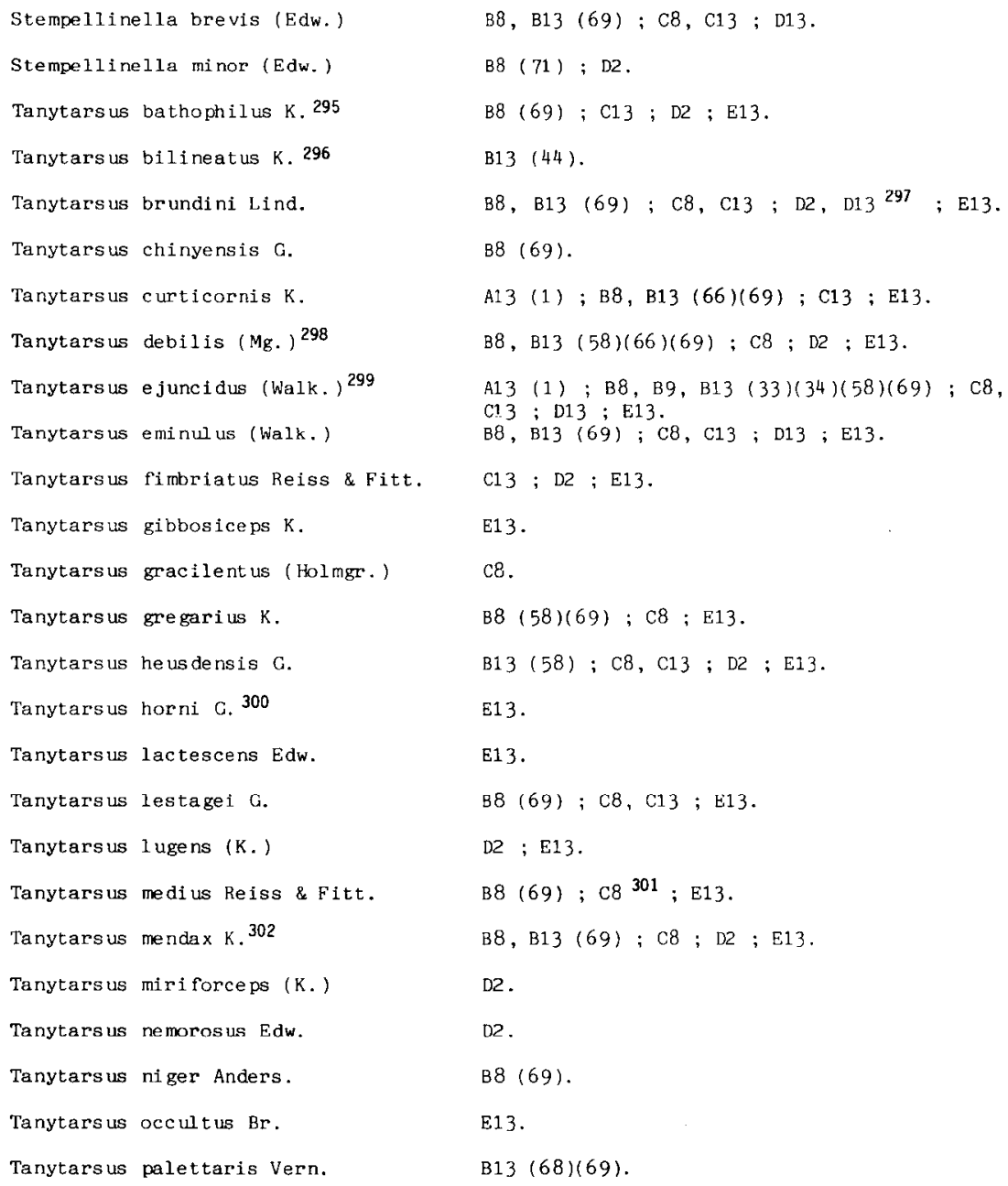


Tanytarsus pallidicornis (Walk.) 303

Tanytarsus palmeni Lind.

Tanytarsus pentamerus $(K . \quad 304$

Tanytarsus quadridentatus $\mathrm{Br}$.

Tanytarsus signatus (v.d.W.)

Tanytarsus sinuatus $G$.

Tanytarsus sylvaticus (v.d.W.)

Tanytarsus tika (Tourenq)

Tanytars us usmaensis Pag.

Tanytarsus verralli $\mathrm{G}$.

Tanytarsus volgensis Miseiko

Virgatanytars us arduennens is (G.)

Virgatanytarsus triangularis (G.)

Zavrelia pentatoma $k$.

TELMATOGETONI NAE (2 espèces)

Telmatogeton pectinatum Deby

Thalassomya frauenfel di Schiner 307
A13 (10)(15); B8, B13 (66)(69).

c8.

E.

E13.

B8 (58); D13.

C13 305 .

D13.

E13.

$\mathrm{B} 8, \mathrm{~B} 13(69) ; \mathrm{E} 13$.

B8, B13 (69).

E13.

$\mathrm{B} 8, \mathrm{~B} 13(66)(69) ; \mathrm{C} 8, \mathrm{C} 13 ; \mathrm{D} 13 ; \mathrm{E} 13$.

$\mathrm{C} 8, \mathrm{C} 13$.

D13.

D1. $3^{306}$.

A13 (7) ; D13 (59) ; E13 ; F3 (19).

\section{Addendum}

\section{Tanytarsus cretensis Reiss 1987}

Signalé par Reiss (1987) dans le Bas-Rhône au sud de Lyon (C 13) au niveau des Centres électronucléaires de Chavanay, St Vulbas et Aramon (Récoltes J. Verneaux).

Reiss (F.). 1987. - Tanytarsus cretensis sp. n., eine neue westpalaearktische Chironomidenart aus Fliessgewässern. Nachrichtenblatt der Bayerischen Entomologen, 36 (1) : 26-30. 


\section{Notes et commentaires}

1. Selon Fittkau \& Reiss (1978), l'appartenance de cette espèce à un genre bien défini n'est pas encore pleinement reconnue. Ashe \& Cranston (1990) la considèrent comme nomen dubium.

2. Martinot (1979). Citation oubliée dans Serra-Tosio (1989b).

3. = ater Macq

4. Gagneur (1976). Citation oubliée dans Laville \& Serra-Tosio (1987)

5. Macquart (1834) : Bordeaux. Citation omise dans Laville \& Serra-Tosio (1987).

6. = costalis $\mathrm{K}$.

7. = obscurus Macq., selon Goetghebuer (1927) et ? = obscurus Macq., selon Goetghebuer (1936).

$8 .=$ goetghebueri $\mathrm{K}$.

9. = septemmaculatus $\mathrm{K}$. ; = bimaculatus $\mathrm{K}$. ; = bitensis $\mathbf{K}$.

10. Selon Serra-Tosio et contrairement à l'opinion de Fittkau (1962), probablement pas synonyme de Monopelopia tenuicalcar (K.).

11. = brevitibialis $\mathrm{G}$.

12. = caliptera $\mathrm{K}$.

13. = tetrasema $\mathrm{K}$.

14. Nomen dubium, selon Ashe \& Cranston (1990).

15. Mentionné sous le nom de Trichotanypus bifasciatus Stgr. (sic !) par Gouin (1936a, 1936b).

16. Cité de France, sans autre mention, par Goetghebuer $(1927,1936)$.

17. = fasciatus Macq. Ce dernier nom est considéré comme nomen dubium par Ashe \& Cranston (1990).

18. Nomen dubium, selon Ashe \& Cranston (1990).

19. = albinervis $\mathbf{K}$.

20. Cité de France, sans autre précision, par Goetghebuer (1927). Peut-être synonyme de Monopelopia tenuicalcar (K.) selon Ashe \& Cranston (1990).

21. Cité de France, sans autre précision, par Goetghebuer $(1927,1936)$.

22. Vaillant (1955, p. 163). Station omise dans SerraTosio (1989b).

23. = iohannseni $\mathrm{K}$. Ce dernier nom est considéré comme nomen dubium par Ashe \& Cranston (1990).

24. Holmière (1988).

25. Vinçon (1987a, 1987b) ; Holmière (1988).

26. Signalé de France, sans autre précision, par Ashe \& Cranston (1990).

27. Cité des Pyrénées par Laville en 1966, mais pas ultérieurement.

28. Holmière (1988).

29. Cité des Pyrénées par Laville (1966), mais considéré ultérieurement (Laville 1980a) comme identification incertaine.

30. = punctulata G. ; = Heptagia sp. A Saunders, 1930.
31. = Heptagia sp. B Saunders, 1930.

32. Holmière (1988).

33. Espèce insuffisamment décrite, à considérer probablement comme nomen dubium.

34. Goetghebuer (1939a) : Lautaret. Citation oubliée dans Serra-Tosio (1989b).

35. = berardensis Ser.-Tos.

36. Holmière (1988).

37. Cité de France, sans autre précision, par Vaillat (1955).

38. = camptoneura $\mathrm{K}$. ; = thienemanni $\mathrm{K}$.

39. Hubault (1927) ; citation omise dans Serra-Tos: (1989b).

40. Sub culicoides Heeg.

41. Holmière (1988).

42. Dans Ashe \& Cranston (1990), Onychodiamesa Pag. est synonyme de Diamesa $\mathbf{M g}$.

43. Cette espece est signalée par Kieffer (1919) du MontCenis, localité des Alpes située à cette époque en territoire italien, mais maintenant en territoire français ! Elle a été omise dans Serra-Tosio (1989b)

44. Holmière (1988).

45. = iberica Ser.-Tos., selon Langton \& Moubayed (1990).

46. = Syndiamesa pubitarsis auct. nec Zett.

47. Aux références indiquées dans Serra-Tosio (1989b) ajouter Martinot (1979).

48. = notata Staeg. ; = pruecox $\mathrm{K}$.

49. = lucidus Staeg.

50. Cité de France, sans autre précision, par Goetghebuer (1932, 1940-50).

51. Holmière (1988).

52. La synonymie concernant cette espèce est particulièrement complexe et embrouillée (cf. Cranston 1984). Sa répartition serait complètement à revoir. Tous les spécimens cités de France sous le nom de Orthocladius bipunctellus (Zett.) correspondent probablement en fait à Orthocladius fuscimanus (K.).

53. Gagneur (1976). Référence oubliée dans Laville \& Serra-Tosio (1987).

54. Cité de France, sans autre précision, par Goetghebuer $(1932,1940-50)$.

55. Holmière (1988).

56. Cite de Grenoble (sub. gr. acuticornis) par Vaillant (1955).

57. Gagneur (1976). Référence oubliée dans Laville \& Serra-Tosio 1987.

58. Nomen dubium, selon Ashe \& Cranston (1990).

59. Hubault (1927). Citation oubliée dans Serra-Tosio (1989b).

60. Cette espece marine n'est connue qu'à l'état d'imago femelle (Kieffer in Mercier, 1923). Son appartenance au genre Chaetociadius n'est pas sûre (Goetghebuer 1940-50). Nomen dubium selon Ashe \& Cranston (1990).

61. ? = marinus Hal,, selon Goetghebuer (1932). 
62. = syzygialis Chevrel ; = bicolor $\mathrm{K}$.

63. ? = celeripes Winn. et nomen dubium selon Ashe \& Cranston (1990).

64. Nomen dubium, selon Ashe \& Cranston (1990).

65. Cité de France, sans autre précision, par Ashe \& Cranston (1990).

66. Espèce citée des Pyrénées par Laville en 1966, mais pas ultérieurement. Considérée comme nomen dubium par Ashe \& Cranston (1990).

67. Holmière (1988).

68. Cité de France, sans autre précision, par Goetghebuer (1939c) et considéré comme nomen dubium par Ashe \& Cranston (1990).

69. Gagneur (1976). Référence oubliée dans Laville \& Serra-Tosio (1987).

70. Cité de France, sans autre précision, par Kieffer (1925 b) et considéré comme nomen dubium par Ashe \& Cranston (1990).

71. = unifasciatus Macq., selon Goetghebuer (1932, 1940-50), donc peut-être présent dans le nord de la France.

72. ? Gazagnes \& Laville (1985) ; Vinçon (1987 a, 1987 b).

73. Holmière (1988).

74. Sub motitatrix L. (synonymie probable, selon Hirvenoja 1973).

75. Hirvenoja \& Moubayed (1989).

76. Cité de France, sans autre précision, par Goetghebuer (1932, 1940-50) et par Hirvenoja (1973), qui se réfèrent tous deux à Macquart.

77. Holmière (1988).

78. Appartenance au genre Cricotopus douteuse. Considéré comme nomen dubium par Ashe \& Cranston (1990).

79. Holmière (1988).

80. = festivus $\mathrm{Mg}$.

81. Cité de France (sub oscillator $\mathbf{M g}$.), sans autre précision, par Goetghebuer (1932, 1940-50).

82. Sub biformis Edw.

83. Gagneur (1976). Citation oubliée dans Laville \& Serra-Tosio (1987).

84. Cité de France, sans autre précision, par Goetghebuer (1932, 1940-50). Mais il s'agit peut-être en fait de $C$. sylvestris (Mg.), dont $C$. ornatus serait, selon Goetghebuer, une simple variété.

85. ? Tourenq (1970), sub oscillator Mg. Référence oubliée dans Serra-Tosio (1989 b).

86. Considéré comme nomen dubium par Ashe \& Cranston (1990).

87. ? = trepidulus $\mathrm{G}$.

88. Holmière (1988).

89. ? = marginatus Macq. ; ? = motitatrix L.

90. Gagneur (1976). Référence oubliée dans Laville \& Serra-Tosio (1987).
91. La citation "France », sans autre précision, par Goetghebuer (1932) fait probablement référence à Macquart (1826, 1834 ; nord de la France).

92. Holmière (1988).

93. Cité de France, sans autre précision, par Goetghebuer (1932).

94. = pseudosimilis $\mathrm{G}$.

95. Holmière (1988).

96. = ephemerae $\mathbf{K}$.

97. = hospita Edw.

98. Holmière (1988).

99. Selon Lehmann (1972), cette espèce n'appartiendrait pas au genre Eukiefferiella et devrait être considérée comme nomen dubium.

100. = potthast $i$ Lehm. Selon Cranston (1975), le nom gracei Edw. est tout à fait valide et ne doit pas être remplacé par potthasti Lehm.

101. = mirabilis Ser.-Tos.

102. Holmière (1988).

103. Selon Goetghebuer (1932, 1940-50), synonyme de hirtipes Macquart, et donc présent dans le nord de la France. Toutes les autres citations françaises de Eurycnemus crassipes (Massif Central, Pyrénées) se rapportent en fait à Buchonomyia thienemanni Fitt.

104. Biarritz (Hirvenoja 1973). Référence omise dans Laville \& Vinçon (1986).

105. = stagnorum $\mathrm{G}_{\text {.; }}=$ maritimus $\mathrm{G}$.

106. ? = marinus Alv., selon Hirvenoja (1973).

107. Sub vitripennis $\mathrm{Mg}$.

108. ? Remmert (1953), sub vitripennis $\mathrm{Mg}$.

109. = halophilus $\mathrm{K}$. et cité sous ce nom de France, sans autre précision, par Goetghebuer (1932; 1940-50, avec répartition reportée par erreur à humeralis !).

110. Jolivet (1973) : côte du Languedoc (Carnon),

111. = intermedia Ser.-Tos.

112. Holmière (1988).

113. Martinot (1979). Citation oubliée dans Serra-Tosio (1989 b).

114. Holmière (1988).

115. Nomen dubium, selon Ashe \& Cranston (1990).

116. = smolandicus $\mathrm{Br}$.

117. Holmière (1988).

118. Selon Ashe \& Cranston (1990), peut-être synonyme de Smittia aterrima (Mg.).

119. = truncorum G.

120. = pusillus Eat.; = interruptus $\mathrm{G}$. : ? = exiguus $\mathbf{G}$.

121. Cité de France, sans autre précision, par Goetghebuer (1940-50).

122. Gagneur (1976). Citation oubliée dans Laville \& Serra-Tosio (1987).

123. Sub exiguus G., nomen dubium et ? = minimus Mg., selon Saether (1990).

124. ? = jemtlandicus $\mathrm{Br}$., ce dernier nom étant considéré comme nomen dubium selon Saether (1990). 
125. Cité des Pyrénées (sub cf. jemtlandicus) par Laville \& Lavandier (1977) et par Vinçon (1987a, 1987b), mais non retenu par Laville \& Vinçon (1986).

126. = prolongatus $\mathrm{K}$.

127. Holmière (1988).

128. = globifer Lundstr.

129. Nomen dubium, selon Saether (1990).

130. Kieffer (1921b) : département des Deux-Sèvres. Espèce non citée dans Laville \& Serra-Tosio (1987). Considérée comme nomen dubium par Ashe \& Cranston (1990).

131. = atratulus Zett.

132. Kieffer (1921b) : département des Deux-Sèvres. Espèce non citée dans Laville \& Serra-Tosio (1987). Considérée comme nomen dubium par Ashe \& Cranston (1990).

133. Cité de France, sans autre précision, par Kieffer (1925b) et considéré par Ashe \& Cranston (1990, sub atractus, sic !) comme nomen dubium.

134. Nomen dubium, selon Ashe \& Cranston (1990).

135. Cité de France, sans autre précision, par Goetghebuer $(1932,1940-50)$.

136. = hygropetricus $\mathrm{K}$., selon Saether (1989).

137. Cité de France, sans autre précision, par Goetghebuer $(1932,1940-50)$.

138. Nomen dubium, selon Ashe \& Cranston (1990).

139. Vinçon (1987a, 1987b).

140. Holmière (1988).

141. L'appartenance de cette espèce au genre Orthocladius est douteuse selon Fittkau \& Reiss (1978). Nomen dubium selon Ashe \& Cranston (1990).

142. Nomen dubium, selon Ashe \& Cranston (1990).

143. Synonyme de Symbiocladius rhithrogenae (Zavr.) selon Codreanu (1939), mais pas selon Goetghebuer (1940-50).

144. Holmière (1988).

145. Probablement synonyme de rivicola K., selon SerraTosio (1989b). Nomen dubium selon Ashe \& Cranston (1990).

146. = sordidellus $K$. nec Zett., selon Goetghebuer (1940-50).

147. Nomen dubium, selon Ashe \& Cranston (1990).

148. ? = fuscimanus K., selon Cranston (1984) et nomen dubium selon Ashe \& Cranston (1990).

149. Holmière (1988).

150. Nomen dubium, selon Ashe \& Cranston (1990).

151. Aux références indiquées dans Serra-Tosio (1989b) ajouter Martinot (1979).

152. Sub obtexens Walk.

153. Holmière (1988).

154. Nomen dubium selon Ashe \& Cranston (1990), mais pas selon Saether (1990).

155. Holmière (1988).

156. Cité de France, sans autre précision, par Vaillant (1955, p. 43).
157. ? = rufiventris $\mathrm{Mg}$.

158. Selon Cranston (1982) et contrairement à l'opinion de Hirvenoja (1973), pas synonyme de skirwithensis Edw.

159. Cité du Massif Central par Verneaux (1968a), mai oublié dans Laville \& Serra-Tosio (1987).

160. Cité des Pyrénées par Laville (1971), mais non retenu dans Laville \& Vinçon (1986).

161. Holmière (1988).

162. = fluviatilis $\mathrm{G}$.

163. Holmière (1988).

164. = curtistylus $\mathrm{G}$. ; = curtistylatus $\mathrm{G}$. ; = torrentium G.

165. Holmière (1988).

166. Station signalée par Wülker (1956) : "Dauphiné. Lac de petit chateaux » (sic !) «Ps. islandicus var. ». Selon Serra-Tosio, aucun lac de ce nom n'existe dans les Alpes du Dauphiné, c'est-à-dire dans les actuels départements de la Drôme, de l'Isère et des HautesAlpes. Il doit s'agir du lac de Petichet (commune de Saint-Théoffrey, département de l'Isère, $920 \mathrm{~m}$ ). autrefois orthographié «Petit Chat ", devenu « petit chateaux » probablement par interprétation abusive des mentions portées sur l'étiquette des spécimens.

167. L'appartenance de cette espèce au genre Psectrocladius n'est pas sûre.

168. = edwardsi $\mathrm{Br}$.

169. = stratiotis $\mathbf{K}$.

170. Gagneur (1976). Citation oubliée dans Laville \& Serra-Tosio (1987).

171. Moubayed (1989).

172. Holmière (1988).

173. = foveatus Edw.

174. = dorieri $\mathrm{G} . ;$ = holosericeus $\mathrm{G}$.

175. = dispar $\mathrm{G}$.

176. Holmière (1988).

177. ? = glabricollis $\mathrm{Mg}$.

178. Holmière (1988).

179. Nomen dubium selon Ashe \& Cranston (1990).

180. ? = foliata K., 1906.

181. Selon Hirvenoja (1973), l'appartenance de cette espèce au genre Smittia est seulement probable. Nomen dubium selon Ashe \& Cranston (1990).

182. Nomen dubium, selon Ashe \& Cranston (1990).

183. Kieffer (1921b) : département des Deux-Sèvres. Espèce omise dans Laville \& Serra-Tosio (1987) et considérée comme nomen dubium par Ashe \& Cranston (1990).

184. Cité de France, sans autre précision, par Goetghebuer $(1932,1940-50)$.

185. Nomen dubium, selon Ashe \& Cranston (1990).

186. Gagneur (1976). Citation oubliée dans Laville \& Serra-Tosio (1987). 
187. Cité de France, sans autre précision, par Cranston (1975).

188. Gagneur (1976). Citation oubliée dans Laville \& Serra-Tosio (1987).

189. = thalassobia G. Ce dernier nom est considéré comme nomen dubium par Ashe \& Cranston (1990).

190. Cité de France, sans autre précision, par Goetghebuer (1940-50).

191. = brevipalpis G., selon Codreanu (1939).

192. Holmière (1988).

193. = tripilatus Edw.

194. Holmière (1988).

195. Cité de la côte française, sans autre précision, par Goetghebuer (1940-50).

196. = clavaticornis $\mathrm{K}$.

197. = fusca $\mathbf{K}$. ; = morosa $\mathbf{E d w}$.

198. Nomen dubium, selon Ashe \& Cranston (1990).

199. Holmière (1988).

200. Espèce appartenant peut-être au genre Smittia, selon Hirvenoja (1973). Nomen dubium selon Ashe \& Cranston (1990).

201. Holmière (1988).

202. Espèce comprise sensu Strenzke (1959). Pour plus de précisions sur son statut, cf. Lindeberg \& Wiederholm (1979)

203. Cité de France, sans autre précision, par Goetghebuer (1928).

204. Nomen dubium, selon Ashe \& Cranston (1990).

205. = halophilus $\mathrm{K}$.

206. Cité de France, sans autre précision, par Goetghebuer (1928).

207. Vinçon (1987a, 1987b).

208. = hexastictus $\mathrm{K}$.

209. Cité de France, sans autre précision, par Goetghebuer $(1928,1937.54)$, probablement par mise en synonymie de $C h$. cingulatus rusticus $\mathrm{Mg}$. avec $C h$. nigroviridis Macq. du nord de la France.

210. = nigroviridis Macq. Pour plus de précisions sur le statut de cette espèce, cf. Lindeberg \& Wiederholm (1979).

211. Nomen dubium, selon Ashe \& Cranston (1990).

212. Gagneur (1976). Citation oubliée dans Laville \& Serra-Tosio (1987).

213. Nomen dubium, selon Ashe \& Cranston (1990).

214. Macquart (1834): France, sans autre précision. Nomen dubium, selon Ashe \& Cranston (1990).

215. Gagneur (1976). Citation oubliée dans Laville \& Serra-Tosio (1987).

216. Nomen dubium, selon Ashe \& Cranston (1990).

217. Cité de France, sans autre précision, par Goetghebuer (1928).

218. = thummi $\mathrm{K}$. Cité de France, sans autre précision, par Goetghebuer (1928, 1937-54).

219. Nomen dubium, selon Ashe \& Cranston (1990).
220. Macquart (1834) : Bordeaux. Citation omise dans Laville \& Serra-Tosio (1987).

221. Les citations de $C h$. salinarius de stations françaises non côtières (Massif Central, Jura, Crau) sont douteuses, car cette espèce est halobionte (cf. Lindeberg \& Wiederholm 1979, p. 103).

222. Remmert (1953), Cheraitia (1984) : littoral du Languedoc. Citations oubliées dans Laville \& SerraTosio (1987).

223. Selon Lindeberg \& Wiederholm (1979), espèce à statut incertain.

224. Nomen dubium, selon Ashe \& Cranston (1990).

225. Considéré comme variété de $C h$. dorsalis par Edwards (1929) et par Goetghebuer (1937-54) et peut-être comme synonyme de cette espèce par Ashe $\&$ Cranston (1990).

226. ? = riparius Mg., selon Goetghebuer (1928). Nomen dubium, selon Ashe \& Cranston (1990).

227. Nomen dubium, selon Ashe et Cranston (1990).

228. Cité de France, sans autre précision, par Goetghebuer $(1928,1937-54)$.

229. Gagneur (1976). Citation oubliée dans Laville \& Serra-Tosio (1987).

230. = atriforceps $\mathrm{G}$.

231. ? = lobiger K. Cité de France, sans autre précision, par Goetghebuer (1928, 1937-54).

232. = pulsus Walk.

233. = falciformis $\mathrm{K}$.

234. Genus dubium, selon Ashe \& Cranston (1990).

235. = dilatatus $\mathrm{G}$.

236. Cité de France, sans autre précision, par Goetghebuer $(1928,1937-54)$.

237. = notabilis Macq. La citation de France, sans autre précision, par Goetghebuer (1928) est probablement erronée, car elle se rapporte sans doute a notabilis que Macquart cite du nord de l'Allemagne (et non de France).

238. Nomen dubium, selon Ashe \& Cranston (1990).

239. Cité de France, sans autre précision, par Goetghebuer (1928).

240. Cité de France, sans autre précision, par Goetghebuer (1937-54).

241. Gagneur (1976). Citation oubliée dans Laville \& Serra-Tosio (1987).

242. Cité de France, sans autre précision, par Goetghebuer $(1928,1937-54)$.

243. ? = foliicola K., selon Ashe \& Cranston (1990).

244. Cité de France, sans autre précision, par Macquart (1834).

245. = pseudosimplex $\mathrm{G}$.

246. = paraderibae Lav. \& Tour.

247. Cité de France, sans autre précision, par Goetghebuer $(1928,1937-54)$.

248. Ajouter : Gagneur (1976), citation oubliée dans Laville \& Serra-Tosio (1987). 
249. = longiforceps $\mathrm{K}$.

250. Cité de France, sans autre précision, par Lehmann (1970b).

251. = schlizensis Ringe.

252. Holmière (1988) ; Moubayed (1990).

253. = obscura $\mathrm{B} r$.

254. = brachylabis Edw.

255. Cité de France, sans autre précision, par Goetghebuer $(1928,1937-54)$

256. Nomen dubium, selon Ashe \& Cranston (1990).

257. ? = albimanus Mg., selon Goetghebuer (1937-54).

258. Nomen dubium, selon Ashe \& Cranston (1990).

259. Gagneur (1976). Citation oubliée dans Laville \& Serra-Tosio (1987).

260. = albimanus, selon Brundin (1949). Mais cette opinion n'est apparemment pas retenue par les auteurs ultérieurs.

261. Nomen dubium, selon Ashe \& Cranston (1990).

262. = vetterense $\mathrm{Br}$.

263. Holnière (1988).

264. ? = testaceum Macq., selon Goetghebuer (1928, 1937-54).

265. Holmière (1988).

266. Nomen dubium, selon Ashe \& Cranston (1990).

267. Holmière (1988).

268. Sub nebulosum (sic !).

269. = pharao $\mathrm{K}$.

270. = prolixitarsis Lundstr.

271. Sub scalaenum Schr.

272. = breviantennatum Chern. Cité de France, sans autre précision, par Goetghebuer (1928, 1937-54).

273. Nomen dubium, selon Ashe \& Cranston (1990).

274. = fasciatus Goet. ; = occultus $\mathrm{K}$. ; = bitensis $\mathrm{K}$.

275. Gagneur (1976). Citation oubliée dans Laville \& Serra-Tosio (1990)

276. = histrio Fab. Cité de France, sans autre précision, par Goetghebuer (1928, 1937-54).

277. Selon Reiss (1969b), ce nom de genre et ce nom d'espèce ne sont pas valides (nomen dubium), car le manuscrit dans lequel Thienemann devait les décrire n'a jamais été publié. D'après Reiss (op, cit.), il pourrait s'agir d'un Chironominae du genre Micropsectra. Selon Ashe \& Cranston (1990), Gowinielia Thien., genre non valide dont pourtant l'espece-type désignée est Krenopsectra acuta (G.), est donc un synonyme de Krenopsectra Reiss.

278. Vaillant (1955). Citation oubliée dans Laville \& Serra-Tosio (1987).

279. Reiss (1969a). Citation oubliée dans Laville \& SerraTosio (1987).

280. L'appartenance de cette espèce au genre Micropsectra est douteuse. Nomen dubium, selon Ashe \& Cranston (1990).

281. Nomen dubium, selon Ashe \& Cranston (1990).
282. Martinot (1979). Citation oubliée dans Serra-Tosio (1989b).

283. Cité de France, sans autre précision, par Goutghebuer (1928) mais pas par Groetghebuer (1937-54,

284. = foliato Lav.

285. Holmière (1988).

286. = praecox auct, nec Wied. in Mg., selon Säweda] (1976).

287. = coracina $\mathrm{K}$.

288. = pyrenaea Fitt.

289. Martinot (1979). Citation oubliée dans Serra-To, (1989b).

290. = atrolineatus $\mathrm{G}$.

291. = boreoalpinus Thien., provenant notamment des Pyrénées françaises, selon Ashe \& Cranston (1990).

292. Nomen dubium, selon Ashe \& Cranston (1990).

293. Gagneur (1976). Citation oubliée dans Laville \& Serra-Tosio (1987).

294. Considéré par Lehmann (1970a) et par Ashe \& Cranston (1990) comme nomen dubium.

295. = tripunctatus Reiss.

296. Considéré par Reiss \& Fittkau (1971) et par Ashe \& Cranston (1990) comme nomen dubium.

297. Holmière (1988).

298. = samboni Edw.

299. = ordinatus $\mathrm{G}$.

300. = nigrocinctus Freem.

301. Gagnc ir (1976). Citation oubliée dans Laville \& Serra-Tosio (1987).

302. = holochlorus Edw., selon Lindeberg (1976). Selon Ashe \& Cranston (1990), l'espèce décrite sous le nom de mendax par Reiss \& Fittkau (1971) correspond en fait à une espèce différente et requiert par consé. quent une dénomination nouvelle.

303. = paschalis $\mathrm{G}$.

304. Espèce douteuse, selon Ashe \& Cranston (1990).

305. Gagneur (1976). Citation oubliée dans Laville \& Serra-Tosio (1987).

306. Deby (1889) : Biarritz. Citation non indiquée dans Laville \& Vinçon (1986).

307. = Scopelodromus isemerinus Chevrel.

\section{Conclusion}

Le total des Chironomidés recensés sur le territoire français et la Corse s'élève actuellement à 132 genres et 598 espèces ainsi répartis :

Genres Espèces

$\begin{array}{lrr}\text { Tanypodinae } & 24 & 59 \\ \text { Podonominae } & 1 & 1 \\ \text { Diamesinae } & 8 & 40 \\ \text { Prodiamesinae } & 3 & 5 \\ \text { Buchonomyinae } & 1 & 1 \\ \text { Orthocladiinae } & 48 & 257\end{array}$




$\begin{array}{lrr}\text { Chironominae } & & \\ \text { Chironomini } & 29 & 142 \\ \text { Pseudochironomini } & 1 & 1 \\ \text { Tanytarsini } & 15 & 90 \\ \text { Telmatogetoninae } & 2 & 2\end{array}$

Ce total, qui approche les 600 espèces, est supérieur aux chiffres des recensements récents proposés pour d'autres pays d'Europe :

$\begin{array}{ll}\text { Espagne + Portugal : } & 327 \text { espèces (Cobo et al. } \\ & 1987 \text {, Reiss 1989) ; } \\ \text { Irlande } & : 347 \text { espèces (Murray \& } \\ & \text { Ashe 1984); } \\ \text { Italie } & : 359 \text { espèces (Rossaro } \\ & 1988) ; \\ \text { Roumanie } & : 405 \text { espèces (Cure 1985) ; } \\ \text { Grande-Bretagne } \quad: 448 \text { espèces (Cranston } \\ \end{array}$

Malgré l'insuffisance notoire des prospections dans certaines régions du Centre et du Nord, on peut considérer la faune française de ces Diptères comme riche et variée puisqu'elle représente près de la moitié $(46 \%)$ des Chironomidés répertoriés de la région paléarctique (Ashe \& Cranston 1990).

Une telle liste, mise à jour depuis les origines des recherches sur ce groupe de Diptères constituera, nous l'espérons, une base pour des travaux comparatifs de biogéographie et d'écologie, puisqu'elle intéresse six zones $(2,3,4,8,9,13)$ de la Limnofauna Europaea.

\section{Travaux cités}

Ashe (P.) \& Cranston (P.S.). 1990. - Family Chironomidae. In : Soos (A.) \& Papp (L.) ed., Catalogue of Palaearctic Diptera, vol. 2, Psychodidae-Chironomidae, Akad. Kiado, Budapest : 113-499.

1. - Bazerque (M.F.), Laville (H.) \& Brouquet (Y.). 1989. Biological quality assesment in two rivers of the northern plain of France (Picardie) with special reference to Chironomid and Diatom indices. Acta Biol. Debr. Oecol. Hung., $3: 29-39$.

2. - Bertrand (H.). 1949. - Capture de Chironomides marins. Bull. Lab. marit, Dinard, 32 : 36-40.

- Brundin (L.). 1949. - Chiromiden und andere Bodentiere der südschwedischen Urgebirgsseen. Rep. Inst. Freshwat. Res. Drottningholm, 30 : 1-914.

3. - Brundin (L.). 1976. - A Neocomian chironomid and Podonominac-Aphroteniinae (Diptera) in the light of phylogenetics and biogeography. Zool. Scripta, 5 : 139.160.

4. - Caspers (H.). 1951. - Rhythmische Erscheinungen in der Fortpflanzung von Clunio marinus (Dipt., Chiron.) und das Problem der lunaren Periodizität bei Organismen. Arch. Hydrobiol. Suppl. $18: 415-594$.
5. - Cheraitia (M.L.) 1984. - Essai d'appreciation de la qualité de quelques milieux stagnants de faible profondeur à partir des macroinvertébrés. Application aux bassins d'épuration par lagunage. Thèse de 3 cycle, Univ. Claude-Bernard Lyon I, 99 p.

6. - Chevrel (R.). 1894. - Sur un diptère marin du genre Clunio Haliday. Arch. Zool. exp. genn., 3 : 583.598.

7. - Chevrel (R.). 1903. - Scopelodromus isemerinus, genre nouveau ex espèce nouvelle de Diptères marins. Arch. Zool. ex. osp. gén., 1 : 1-29.

- Cobo (F.) et al, 1987, - Inventario faunistico de los Qujronomidos conocidos de la Peninsula iberica. Alquibla : 56-65.

- Codreanu (R.). 1939. - Recherches biologiques sur un Chironomide, Symbiocladius rhithrogence (Zav.), ectoparasite "cancérigène " des Ephémères torrenticoles. Archs Zool. exp. gén., $81: 1-283$.

8. - Comas (M.). 1927. - Sur les métamorphoses de Prodiamesa notata Meigen (Chironomidae), Bull. Soc. zool. Fr., 52 : 174-178.

- Cranston (P.S.). 1975. - Corrections and additions to the list of British Chironomidae (Diptera). Ent. Mon. Mag., $110: 87-95$.

- Cranston (P.S.). 1976. - Chironomidae. In : A Check list of British insects (ed. by Kloet and Hincks). Royal Ent. Soc. London, 11 : 12-21

- Cranston (P.S.). 1984. - The taxonomy and ecology of Orthocladius (Eudactylocladius) fuscimanus (Kieffer), a hygropetric chironomid (Diptera). Journ. Nat. Hist., $18: 873-895$.

- Cure (V.). 1985. - Chironomidae (Diptera-Nematocera) aus Rumänien unter besonderer Berücksichtigung jener aus dem hydrographischen Einzugsgebiet der Donau. Arch. Hydrobiol. Suppl. 68 : 163-217.

- Deby (J.). 1889. - Description of a new dipterous insect, Psammathiomyia pectinata. J.R. microsc. Soc. B. : 180-186.

9. - Delettre (Y.). 1977. - Contribution à l'étude des populations larvaires de Parasmittia sp. carinoto Strenzke affinis (Diptera, Chironomidae) dans une formation pionnière de la lande armoricaine. Rev. Ecol. Biol. Sol, 13 : 579-594.

10. - Delettre (Y.). 1984. - Recherche sur les Chironomides (Diptera) à larves édaphiques. Biologie, écologie, mécanismes adaptatifs. Thèse doctorat d'Etat, Univ. de Rennes I : 310 p.

11. - Delettre (Y.). 1986 a. - La colonisation de biotopes multiples : une alternative à la résistance in situ aux conditions météorologiques défavorables. Cas de Limnophyes minimus ( $\mathrm{Mg}$ ), Diptère Chironomidae à larves édaphiques des landes armoricaines. Rev. Ecol. Biol. Sol, 23 : 29-38.

12. - Delettre (Y.). 1986 b. - Adaptative strategies in terrestrial Chironomidae. First Internat. Congr. Dipterology, Budapest, 1986. Workshop D : Chironomidae : $9 \mathrm{p}$.

13. - Delettre (Y). 1988 a. - Flux d'évaporation corporelle et résistance à la dessiccation chez les larves de quelques Chironomidae terrestres. (Diptera). Rev. Ecol. Biol. Sol, $25: 129-138$. 
14. - Delettre (Y.). 1988 b. - Chironomid wing length, dispersal ability and habitat predictability. Holarctic Ecology, $11: 166-170$.

15. - Delettre (Y.). 1989 a. - Influence de la durée et de l'intensité de l'assèchement sur l'abondance et la phénologie des Chironomides (Diptera) d'une mare semipermanente peu profonde. Arch. Hydrobiol., 114 : 383-399.

16. - Delettre (Y.). 1989 b. - Response of terrestrial Chironomid communities to fire : an experimental study on heathlands in Brittany (France). Acta Biol. Debr. Oecol. Hung., 3 : 101 -109.

17. - Delettre (Y.) \& Bailliot (S.). 1977. - Sur la résistance de larves de Chironomidae Orthocladiinae à l'asséchement du sol. C.R. Acad. Sci. Paris, 284 : 1717-1719.

18. - Duviard (D.) \& Thréhen (P.). 1981. - Modifications du peuplement d'arthropodes d'une lande à ajoncs de Bretagne centrale induites par l'épandage de déchets ménagers broyés. Oecol. Applic., 2 : 317-337.

19. - Edwards (F.W.). 1928. - The nematocerous Diptera of Corsica. Diptera, 4 : 157-189.

- Edwards (F.W.). 1929. - British non-biting midges (Diptera, Chironomidae), Trans. R. ent. Soc. Lond., 77 : 279-430.

20. - Feuillade (J.). 1965. - Contribution à l'étude d'un lac de barrage : le lac de Vézins (Manche). Thèse Univ. Caen : $176 \mathrm{p}$.

21. - Fittkau (E.J.). 1962. - Die Tanypodinae (Diptera, Chironomidae). Die Tribus Anatopyniini, Macropelopiini und Pentaneurini. Abh. Larvalsyst. Insekten, $6: 1-453$.

Fittkau (E.J.) \& Reiss (F.). 1978. - Chironomidae. In : Illies (J.), Limnofauna Europaea, 2. Aufl. : 404-440, G. Fischer Verlag, Stuttgart.

22. - Gadeau de Kerville (H.). 1898. - Recherches sur les faunes marine et maritime de la Normandie, $2^{*}$ voyage 1818 . Bull. Soc. Amis Sci. nat. Rouen 1897 : 359-372.

- Gagneur (J.). 1976. - Etude des Diptères du Lot et étude de la retenue de Cajarc. Thèse de $3^{\mathrm{e}}$ cycle, Univ. Toulouse : $195 \mathrm{p}$

23. - Gazagnes (G.) \& Laville (H.). 1985, - Etude faunistique des Chironomidés (Diptera) de la haute Neste d'Aure (Pyrénées centrales) : impact des aménagements hydroélectriques. Annis Limnol., 21 : 149-159.

24. - Geoffroy (E.L.). 1785. - Entomologia Parisiensis. Vol. 1. A.F. Fourcroy (éd.), Paris: $1-544$ p.

25. - Giard (A.). 1898. - Sur l'appareil tracheen de Chunio marinus Haliday, C.R. Ass. fr, Avanc, Sci., $26: 299-300$.

26. - Giard (A.). 1904. - Quelques mots sur l'Hydrobaenus lugubris Fries (Dipt.). Bull. Soc, ent. Fr., 9 : 164-165.

27. - Goetghebuer (M.). 1927. - Diptères (Nèmatocères). Chironomidae Tanypodinae. Faune Fr., $15:$ 1-83.

- Goetghebuer (M.). 1928. - Diptères (Nématocères). Chironomidae. III. Chironomariae, Faune Fr., 5 : 1-174.

28. - Goetghebuer (M.). 1932. - Diptères (Nématocères). Chironomidae. IV. Orthocladiinae, Corynoneurinae, Clunioninae, Diamesinae. Faune Fr., 23 : 1-204.

29. - Goetghebuer (M.). 1936. - Tendipedidae (Chironomidae). a) Subfamilie Pelopiinac (Tanypodinae). A. Die Imagines. In : Lindner (E.) ed., Die Fliegen der palaearktischen Region, 13 b : 1-50.
Goetghebuer (M.). 1937-1954. - Tendipedidae (Chironomidae). b) Subfamilie Tendipedinae (Chironominae). A. Die Imagines. In : Lindner (E.) ed., Die Fliegen der palaearktischen Region, $13 \mathrm{c}: 1-138$.

- Goetghebuer (M.). 1939 a. - Tendipedidae (Chironomidae). c) Subfamilie Diamesinae. A. Die Imagines. In : Lindner (E.) ed., Die Fliegen der palaearktischen Region, $13 \mathrm{~d}: 128$.

30. - Goetghebuer (M.). 1939 b. - Tendipedidae (Chironomidae). d) Subfamilie Podonominae, A. Die Imagines. In : Lindner (E.) ed., Die Fliegen der palaearktischen Region, 13 e : $1-5$.

- Goetghebuer (M.). 1939 c. - Tendipedidae (Chironomidae). e) Subfamilie Corynoneurinae. A. Die Imagines. In: Lindner (E.) ed., Die Fliegen der palaearktischen Region, $13 \mathrm{f}: \mathrm{l}-14$.

31. - Goetghebuer (M.). 1940-1950. - Tendipedidae (Chironomidae). f) Subfamilie Orthocladiinae. A. Die Imagines. In : Lindner (E.) ed., Die Fliegen der palaearktischer Region, $13 \mathrm{~g}: 1-208$.

32. - Goetghebuer (M.). 1944. - Ceratopogonidae et Chironomidae nouveaux ou peu connus d'Europe (douzième note), Biol. Jaarb., 11 : 35-44.

33. - Gouin (F.) 1936 a. - Quelques Chironomides d'Alsace et de Lorraine. 1. Bull. Ass. philomath. Als. Lorr., 8 : 187-192.

34. - Gouin (F.). 1936 b. - Métamorphose de quelques Chironomides d'Alsace et de Lorraine avec la description de trois espèces nouvelles par M. Goetghebuer. Revue fr. Ent., 3: 151-173.

35. - Gouin (F.). 1937 a. - Chironomides de l'Alsace et de la Lorraine (Dipt. Némat.). II. Bull. Ass. philomath. Als. Lorr., 8 : 309-312.

36. - Gouin (F.) 1937 b. - Métamorphoses de quelques Chironomides. Revue Fr. Ent., 4 : 53-60.

37. - Gouin (F.). 1955. - Deux Chironomides nouveaux pour la faune de l'Alsace. Bull. Ass, philomath. Als. Lorr., $9: 161$.

38. - Gouin (F.). 1956. - Parametriocnemus stylatus K. et Parametriocnemus arciger $\mathrm{K}$. (Diptères, Némat, Chironomidae), deux espèces ou deux variétés ? Bull. Ass. philomath. Als. Lorr, 9 : 194-198.

- Hirvenoja (M.). 1973. - Revision der Gattung Cricocotopus van der Wulp und ihrer Verwandten (Diptera, Chironomidae). Ann. zool. fenn., $10:$ 1-363.

Hirvenoja (M.) \& Moubayed (Z.). 1989. - The adults and preimaginal stages of continental populations of $\mathrm{Cr}$. cotopus (Cricotopus) beckeri Hirvenoja (Diptera, Chironomidae). Annts Limnol., $25: 251-254$.

- Holmière (D.). 1988, - La dérive des invertébrés aquatiques sur la moyenne et basse Neste d'Aure (HautesPyrénées). Impact des aménagements hydro-électriques. Thèse Doctorat $3^{e}$ cycle, Spécialité Ecologie, Université Toulouse III (France) : $115 \mathrm{p}$.

39. - Hubault (E.). 1927. - Contribution à l'étude des invertébrés torrenticoles. Bull. biol. Fr. Beig. Suppl. 9 : 159-164. 
Illies (J.) ed. 1978. - Limnofauna Europaea. G. Fischer Verlag, 2nd ed. : $532 \mathrm{p}$.

- Jolivet (P.). 1973. - Le problème des Chironomides (Diptères Nématocères) dans le Languedoc-Roussillon. Vie et Milieu, $23: 269-290$.

40. - Kieffer (J.J.). 1898, - Description d'un Diptère sous-marin recueilli aux Petites-Dalles (Seine Inférieure). Bull. Soc. ent. Fr., 3 : 105-108.

41. - Kieffer (J.J.) 1899 a. - Observations sur le groupe Chironomus avec description de quelques especes nouvelles. Ann. Soc. ent. Fr., $68: 821-830$.

42. - Kieffer (J.J.) 1899 b. - Description d'un nouveau genre et tableau des genres europeens de la famille des Chironomides (Dipt.). Bull. Soc. ent. Fr., 4 : 66-70.

43. - Kieffer (J.J.). 1900. - Description d'une nouvelle espèce de Diptère marin de la famille des Chironomides $(\mathrm{Cl}$ nio bicolor), et renseignements sur cette espèce, découverte par M. Henri Gadeau de Kerville dans l'anse de Saint-Martin (côte septentrionale du département de la Manche) et trouvée par $\mathbf{M}$. René Chevrel à Saint-Briac (Ille-et-Vilaine). Bull. Soc. Amis Sci. nat., P.V. séance du 8.11.1900:72-73.

44. - Kieffer (J.J.). 1906. - Description de nouveaux diptères nématocères d'Europe. Ann. Soc. scient. Brux., 30 : 311-348.

45. - Kieffer (J.J.) 1909. - Diagnoses de nouveaux Chironomides d'Allemagne. Bull. Soc. Hist. nat. Metz, 26 : 37-56.

46. - Kieffer (J.J.). 1918. - Beschreibung neuer, auf Lazarettschiffen der östlichen Kriegsschauplatzes und bei Ignalino in Litauen von Dr. W. Horn gesammelter Chironomiden, mit Übersichtstabelien einiger Gruppen von paläarktischen Arten (Dipt.). Ent. Mitt., $7:$ 35-53, 94-110, 163-170, 177-188.

- Kieffer (J.J.). 1919. - Chironomides d'Europe conservés au Musee National Hongrois de Budapest. Ann. hist.nat. hung., $17: 1-160$.

47. - Kieffer (J.J.) 1921 a. - Neue Chironomiden aus Mitteleuropa. Arch. Hydrobiol. Suppl. 2 : 785-808.

- Kieffer (J.J.) 1921 b. - Chironomides nouveaux ou peu connus de la région paléarctique. Bull. Soc. Hist. nat. Metz, 29 : 51-109.

48. - Kieffer (J.J.). 1922. - Chironomides nouveaux ou peu connus de la région paléarctique. Ann. Soc. scient. Brux., $42: 71-128,138-180$.

49. - Kieffer (J.J.). 1924. - Chironomides nouveaux ou rares de l'Europe centrale. Bull. Soc. Hist, nat. Metz, 30 : 11-110.

50. - Kieffer (J. J.). 1925 a. - Nouveaux représentants de la tribu des Orthocladiariae. Iere partie. Ann. Soc. scient. Brux., 44 : 382-388.

- Kieffer (J.J.). 1925 b. - Deux genres nouveaux et plusieurs especes nouvelles du groupe des Orthocladiariae. Ann. Soc. scient. Brux., 44 : 555-556.

51. - Kieffer (J.J.). 1925 c. - Quatre nouveaux représentants du genre Thienemanniella Kieff. (Dipt. Chironomides). Bull. Soc. ent. Fr., $30: 285$.

52. - Kieffer (J.J.) \& Thienemann (A.). 1908. - Neue und bekannte Chironomiden und ihre Metamorphose. $Z$. wiss. Insektbiol., 4: 1-10, 33-39, 78-84, 124-128, $184-190,214-219,256-259,277-286$.
- Langton (P.H.) \& Moubayed (Z.). 1990 . - Redescription of the pupal exuviae of Potthastio montium Edwards (= iberica Serra-Tosio syn. nov.) (Diptera : Chironomidae). Br. J. Ent. nat. Hist., $3: 135-137$.

- Laville (H.). 1966. - Chironomides du massif de Néouvielle (Pyrénées centrales) (Diptères). Annls Limnol., $2: 203-216$

- Laville (H.). 1971. - Recherches sur les Chironomides (Diptera) lacustres du massif de Néouvielle (HautesPyrénées). Première partie : systématique, écologie, phénologie. Annls Limnol, 7 : 173-332.

- Laville (H.). 1980 a. - Inventaire 1980 des Chironomides (Diptera) connus des Pyténées. Ann's Limnol, 16 : 211-223.

- Laville (H.). 1980 b. - Inventaire des Chironomides connus des Pyrénées en 1976. Acta Univ. Carol.Biologica 1978 : 103-113.

- Laville (H.) \& Lavandier (P.). 1977. - Les Chironomides (Diptera) d'un torrent pyrénéen de haute montagne : l'Estaragne. Annls Limnol., 13 : 57-81.

- Laville (H.) \& Serra-Tosio (B.). 1987. - Chironomides (Diptera) du Massif Central et des basses régions avoisinantes. Annls Limnol., 23 : 135-145.

- Laville (H.) \& Vinçon (G.). 1986. - Inventaire 1986 des Chironomides (Diptera) connus des Pyrènées. Annls Limnol., $22: 239-251$.

- Lehmann (J.). 1970 a. - Revision der europäischen Arten (Imagines $\sigma \sigma$ und Puppen $\sigma \sigma$ ) der Gattung Rheotanytarsus Bause (Dipt., Chironomidae). Zool. Anz., $185: 344-378$.

- Lehmann (J.). 1970 b. - Revision der europäischen Arten (Imagines $\sigma \circ$ ) der Gatuung Parachironomus Lenz (Diptera, Chironomidae). Hydrobiologia, 33 : 129-158.

- Lehmann (J.). 1972. - Revision der europäischen Arten (Puppen $\sigma \sigma$ und Imagines $\sigma \sigma$ ) der Gattung Eukiefferiella Thienemann. Beitr. Ent., 22 : 347-405.

- Lindeberg (B.). 1976. - Taxonomy of some species of the Tanytarsus eminulus group (Dipt., Chironomidae). Ann. zool. fenn., $42: 47-53$.

- Lindeberg (B.) \& Wiederholm (T.). 1979. - Notes on the taxonomy of European species of Chironomus (Diptera, Chironomidae), Ent, scand. Suppl. $10: 99-116$.

53. - Macquart (J.). 1826. - Insectes Diptères du Nord de la France. I. Tipulaires. Lille : 175 p.

54. - Macquart (3.). 1834. - Histoire Naturelle des Insectes. Tome 1.2. Diptères. Paris : $578 \mathrm{p}$.

- Martinot (J.P.J. 1979. - Ecologie et gestion piscicole des lacs de haute altitude du Pare National de la Vanoise. Thèse de 3 cycle, Univ. Scientif. et Méd., Grenoble : $127 \mathrm{p}$.

55. - Mercier (L.). 1920. - Diptères marins et maritimes de la côte du Calvados. Annls Soc. ent. Belg., 60 : 206-208.

56. - Mercier (L.). 1921. - Essai sur la constitution de la faune des Diptères de la côte du Calvados. Congrès de I'A.F.A.S., Rouen 1921 : 643-647.

57. - Mercier (L.). 1923. - Diptères de la côte du Calvados. IVe liste. (Avec la description de deux espèces nouvelles et d'une variété nouvelle de Chironomides marins par $\mathbf{J} . \mathbf{J}$. Kieffer). Ann. Soc. ent. Beig., $63:$ 9-20. 
- Moubayed (Z.). 1989. - Description de Pseudorthocla-

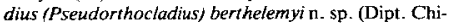
ronomidae, Orthocladinae). Bull. Soc. Hist. Nat. Tou" louse, $125: 27-29$.

- Moubayed (Z.). 1990. - Description of the pupal exuviae of Paracladopelma mikiana G. (Dipt. Chironomidae). Bull. Soc. Hist. Nat. Toulouse, 126: 61-63.

- Murray (D.A.) \& Ashe (P.). 1984. - An inventory of the Irish Chironomidae (Diptera). Mem. Amer. ent. Soc., $34: 223-233$.

- Reiss (F.). 1969 a. - Revision der Gattung Micropsectra Kieff., 1909 (Diptera, Chironomidae). 1. Die attenuataGruppe der Gattung Micropsectra. Beschreibung 5 neuer Arten aus Mitteleuropa und Nordafrika. Dt. ent. $Z$., $16: 431-449$.

- Reiss (F.). 1969 b. - Krenopsectra fallax gen. n. sp. n. (Diptera, Chironomidae) aus den Alpen und Pyrenäen. Ann. zool. fenn., $6: 435-442$.

- Reiss (F.). 1989. - Erster Beitrag zur Chironomidenfauna Portugals (Diptera, Chironomidae). NachrBl. bayer. Ent., 38 : 46-50.

58. - Reiss (F.) \& Fittkau (E.J.). 1971. - Taxonomie und Ökologie europäisch verbreiteter Tanytarsus-Arten (Chironomidae, Diptera). Arch. Hydrobiol. Suppl. 40: 75-200.

59. - Remmert (H.). 1953. - Les Diptères des côtes méditerranéennes de France et d'Espagne. Vie et Milieu, 4 : $540-546$.

- Rossaro (B.). 1988. - A contribution to the knowledge of Chironomids in Italy. Spixiana, Suppl., $14: 191-200$.

- Saether (O.A.). 1989. - Metriocnemus van der Wulp : a new species and a revision of species described by Meigen, Zetterstedt, Staeger, Holmgren, Lundström and Strenzke (Diptera, Chironomidae). Entomol. Scand., $19: 393-430$.

- Saether (O.A.). 1990. - A review of the genus Limnophyes Eaton from the Holarctic and Afrotropical regions (Diptera : Chironomidae, Orthocladiinae). Ent. Scand. Suppl, $35: 1-139$.

60. - Saunders (L.G.). 1930. - The larvae of the genus Heptagia, with description of a new species (Diptera : Chironomidae). Entomol. Mon. Mag., $66: 209-214$.

- Sawedal (L.). 1976. - Revision of the notescens-group of the genus Micropsectra Kieffer, 1909 (Diptera : Chironomidae). Ent. Scand., $7:$ 109-144.

61. - Serra-Tosio (B.). 1981. - Contribution à l'étude du genre Parorthocladius Thien. (Dipt. Chironomidae). Bull. Soc. ent. France, $86: 217-223$.

62. - Serra-Tosio (B.) 1989 a. - Revision des espèces ouestpaléarctiques et néarctiques de Boreoheptagyia avec des clés pour les larves, les nymphes et les imagos (Diptera, Chironomidae). Spixiana, $11: 133-173$.

- Serra-Tosio (B.). 1989 b. - Chironomides (Diptera) des Alpes françaises et des basses régions avoisinantes. Annls Limnol., 25 : 159-175.

- Strenzke (K.). 1959. - Revision der Gattung Chironomus Meig. I. Die Imagines von 15 norddeutschen Arten und Unterarten. Arch. Hydrobiol., $56:$ 1-42.
63. - Tôrres (G.) 1983. - Ecologie des Chironomidae du lac de Créteil (Val-de-Marne, France). Thèse doctorat d'état, Univ. Paris VII : $187 \mathrm{p}$.

Tourenq (J.N.). 1970, - Compléments à l'inventaire des Chironomides de Camargue. Annis Limnol, 6 : 363-370.

64. - Vaillant (F.). 1955. - Recherches sur la faune madicole de France, de Corse et d'Afrique du Nord. Mém. Mus. Hist. nat. Paris (A), $11: 1258$.

65. - Verneaux (J.). 1968 a. - Contribution à l'étude des Chironomides (Diptères Nérnatocères) de la région de Besseen-Chandesse (Puy-de-Dôme). Annls Stn. biot. Besseen-Chandesse, $3: 115-146$.

66. - Verneaux (J.). 1968 b. - Contribution à l'étude d'une petite rivière de plaine, les Doulonnes (Jura). Référence particulière aux Diptères Chironomides. Remarques écologiques. Annis scient. Univ, Besançon (3) $4: 29-40$.

67. - Verneaux (J.). 1969. - Tanytarsus patettaris $\mathrm{n}$. $\mathrm{sp}$. : une nouvelle espèce de Tanytarsini (Diptera, Chironomidae). Description de l'imago $\sigma$ et des stades préimaginaux. Données écologiques. Annls scient. Univ. Besancon (3) $5: 3.13$.

68. - Verneaux (J.). 1970. - Conchapelopia fascigera n. sp. : une nouvelle espece de Tanypodinae (Diptera, Chironomidae). Description de l'imago ơ et données écologiques. Annis scient. Univ. Besançon (3) $6: 59-64$.

69. - Verneaux (J.) \& Vergon (J.P.). 1974. - Faune dulçaquicole de Franche-Conté. Sixième partie : lẹs diptères chjronomides. Annls scient. Univ. Besancon (3) 11 : 179-198.

70. - Vinçon (G.). 1987 a. - Comparaison de la faune benthique des vallées d'Aure et d'Ossau, en vue de l'élaboration d'une méthodologie de surveillance des cours d'eau de montagne. Thèse de Docteur-Ingénieur, Univ. Toulouse : $381 \mathrm{p}$.

- Vinçon (G.). 1987 b. - Etude hydrobiologique de la vallée d'Ossau (Pyrénées-Atlantiques). II. Le milieu et la structure du peuplement benthique. Annis Limnol. 23 : 225-243.

71. Wasson (J.G.). 1984. - L'utilisation des peuplements larvaires de Chironomidae (Diptera) pour la diagnose écologique des lacs : essai d'application aux deux lacs de Clairvaux (Jura, France). Revue fr. Sci. Eau, 3 395-408.

72. - Wülker (W.). 1956. - Zur Kenntnis der Gattung Psectrocladius Kieff. (Dipt., Chironom.). Individuelle Variabilität, Grenzen und Möglichkeiten der Artentrennung. Ökologie und Verbreitung. Arch. Hydrobiol. Suppl., 24: 1-66.

73. - Wülker (W.) 1958. - Die Bedeutung der Chironomiden für die limnologisch-tiergeographische Charakterisierung des Hochschwarzwaldes. Verh. int. Verh. Limnol., 13 : 805.813.

74. - Wülker (W.). 1959. - Diamesarien-Studien (Dipt., Chironomidae) im Hochschwarzwald. Arch. Hydrobiol. Suppl. 24 : 338-360. 